\title{
Modeling the hydro-mechanical responses of strip and circular punch loadings on water-saturated collapsible geomaterials
}

\author{
WaiChing Sun $\cdot$ Qiushi Chen $\cdot$ Jakob T. Ostien
}

Received: 28 January 2013/ Accepted: 13 September 2013/Published online: 22 November 2013

(C) Springer-Verlag (outside the USA) 2013

\begin{abstract}
A stabilized enhanced strain finite element procedure for poromechanics is fully integrated with an elasto-plastic cap model to simulate the hydro-mechanical interactions of fluid-infiltrating porous rocks with associative and non-associative plastic flow. We present a quantitative analysis on how macroscopic plastic volumetric response caused by pore collapse and grain rearrangement affects the seepage of pore fluid, and vice versa. Results of finite element simulations imply that the dissipation of excess pore pressure may significantly affect the stress path and thus alter the volumetric plastic responses.
\end{abstract}

Keywords Bearing capacity $\cdot$ Cap plasticity $\cdot$ Excess pore pressure $\cdot$ Hydro-mechanical coupling .

Poromechanics $\cdot$ Stabilized procedure

\section{Introduction}

The fully coupled diffusion-deformation processes occurring within porous media, such as sand, clay, and rock, are

W. Sun $(\square)$

Department of Civil Engineering and Engineering Mechanics, Columbia University, 500 West 120th Street, New York, NY 10027, USA

e-mail: wsun@columbia.edu

Q. Chen

Glenn Department of Civil Engineering, Clemson University, 101 Sikes Avenue, Clemson, SC 29634, USA

J. T. Ostien

Mechanics of Materials Department, Sandia National

Laboratories, 7011 East Avenue, Livermore, CA 94550, USA of interest to numerous geotechnical engineering applications. The presence of fluid inside the pores and in between the interconnected grains may induce excess pore pressure, limit volumetric deformation, and introduce rate dependence to the mechanical response of the solid skeleton due to the transient nature of fluid diffusion $[14,31]$. Since pore fluid flow may profoundly change the mechanical response of porous media, it is important to take it into account to ensure the sufficiency of engineering designs.

The fluid-solid interaction in porous media is often viewed in the context of mixture theory, in which one or multiple fluids in the connected pore network and a solid skeleton are homogenized as constituents of a continuum mixture $[8,14,31]$. The resulting boundary value problem leads to a two-way coupled system involving at least two balance equations: one to characterize the deformation of the solid skeleton, and one for the mass balance of the pore fluid.

There is an extensive body of literature focusing on finite element analysis of such a coupled system for geotechnical engineering applications $[11,22,28,36,44,51]$. Some of these efforts incorporate critical state plasticity models to capture shear failure and strain localization underneath the foundation. This type of shear failure may be accompanied by various amounts of plastic dilation. Nevertheless, geomaterials, such as high-porosity rock and salt, are vulnerable to both shear and compaction failure. In addition to accumulating plastic dilation due to microcrack growth, grain rotation, and sliding, these materials may exhibit significant inelastic compaction due to pore collapse or grain crushing when the confining pressure is sufficiently high. This distinct micro-mechanical feature not only affects the macroscopic mechanical behavior, but also the interaction between the mechanical and the hydraulic responses. While this plastic compaction can be 
captured by critical state soil models, the cap plasticity model used in this study, if calibrated carefully, may provide more accurate predictions due to the usage of separate evolution equations to capture different mechanisms.

To capture the inelastic compactive behavior of fully saturated rock, we incorporate a cap plasticity model that uses a pressure-dependent shear yielding/failure surface with a hardening compactive elliptical cap to capture the pore collapse. For cap models with non-smooth intersection between the shear yield surface and the compactive cap (e.g. [16, 33]), difficulties may arise from the singularities induced by the non-smoothness of yield surfaces at the intersection. This issue must be addressed by special treatments, which often introduce severe algorithmic and numerical complexities [23]. To overcome this issue, smooth variations of the traditional cap model have been proposed by Borja [10], Dolarevic and Ibrahimbegovic [17], Fossum and Fredrich [18], Grueschow and Rudnicki [21], Swan and Seo [46]. In this paper, we adopt the smooth variation of a three-invariant cap model proposed in Fossum and Fredrich [18] and subsequently implemented and analyzed in Foster et al. [19], Regueiro and Foster [30]. This model features a material state that may move freely from compactive to shear-dominated dilative deformation. The third stress invariant is introduced to capture the difference in strength of geomaterials observed in triaxial compression and extension tests.

While using the same finite element space for displacement and pore pressure is convenient, equal-order spatial discretizations may cause spurious oscillations in pore pressure near the undrained limit due to the lack of the inf-sup condition $[44,51]$. To satisfy the inf-sup condition, one often uses finite dimensional spaces spanned by different basis functions to interpolate the displacement and pore pressure fields. However, the usage of multiple basis functions increases the computational cost and leads to difficulties in post-processing the finite element simulations. Another unrelated but equally important issue is the volumetric locking caused by the incompressibility of the solid skeleton itself. This may occur in granular materials approaching critical state and for soft tissues with little or no compressibility. This volumetric locking can also be cured by introducing an extra degree of freedom for hydrostatic stress to model the volumetric response of the skeleton. Nevertheless, the extra degree of freedom used to cure volumetric locking also causes an additional computational cost and may lead to other stability issues $[13,53]$.

To address these two issues described above, we incorporate an inf-sup stabilization technique (for spurious modes near the undrained limit) along with an assumed strain scheme (for volumetric locking) such that (1) both the displacement and the pore pressure fields may span the same basis functions without spurious oscillations and (2) the resultant finite element solution is locking-free and stable. The formulation is then integrated with a cap-surface model to simulate the fully coupled deformationdiffusion process exhibited in porous rock. By integrating the cap-surface model with the stabilized hydro-mechanical scheme, we study how inelastic compaction affects the hydraulic response of collapsible porous media and predict whether the presence of pore fluid would delay or promote inelastic compaction.

An outline of the paper is as follows. Section 2 reviews the balance laws that characterize the fully coupled deformation-diffusion process and describes the numerical techniques used to stabilize the accompanying finite element solutions. Section 3 outlines the constitutive model for rocks and granular materials vulnerable to pore collapse. Section 4 discusses stress-induced permeability changes. Numerical results are presented in Sect. 5, and conclusions are drawn in Sect. 6.

As for notations and symbols, bold-faced letters denote tensors; the symbol ' $'$ ' denotes a single contraction of adjacent indices of two tensors (e.g. $\boldsymbol{a} \cdot \boldsymbol{b}=a_{i} b_{i}$ or $\boldsymbol{c} \cdot \boldsymbol{d}=c_{i j} d_{j k}$ ); the symbol ' $:$ ' denotes a double contraction of adjacent indices of tensor of rank two or higher (e.g. $\boldsymbol{C}: \boldsymbol{\epsilon}^{\boldsymbol{e}}=C_{i j k l} \epsilon_{k l}^{e}$ ); the symbol ' $\otimes$ ' denotes a juxtaposition of two vectors (e.g. $\boldsymbol{a} \otimes \boldsymbol{b}=a_{i} b_{j}$ ) or two symmetric secondorder tensors (e.g. $\boldsymbol{\alpha} \otimes \boldsymbol{\beta}=\alpha_{i j} \beta_{k l}$ ).

\section{Formulation of poromechanics problems}

In this section, we present the formulation of the poromechanics problem in the infinitesimal regime. We first review the small strain theory with compressible solid and fluid constituents. Then, we introduce the numerical schemes employed to address the numerical deficiencies, particularly the volumetric locking and spurious oscillation in the pore pressure field.

\subsection{Brief review of poromechanics theory}

The central premise of the poromechanics continuum theory is the idea that the total stress, $\boldsymbol{\sigma}$, experienced by a porous medium is the sum of two components. The first component comes from the stress induced by deformation and re-arrangement of the solid grains that form the solid skeleton (the effective stress $\boldsymbol{\sigma}^{\prime}$ ). The second component is the pore pressure $p$, i.e.,

$\boldsymbol{\sigma}=\boldsymbol{\sigma}^{\prime}-B p \boldsymbol{I}$.

Here $B$ is a scalar parameter and the effective stress is equivalent to the stress the solid skeleton would have experienced if the solid skeleton underwent the same deformation without the presence of pore fluid. The scalar 
parameter $B$ has been interpreted from a variety of viewpoints and, as a result, there are multiple effective stress definitions. A common form of the effective stress is from Terzaghi et al. [47] where he deduces that $B$ is a coefficient that depends on the effective surface area of the grains. Skempton [37] performed experiments on soils to measure the range of $B$ and argue on empirical grounds that it equals 1 if both constituents are incompressible but can be much less for rocks composed of deformable grains. Nur and Byerlee [27] consider the porosity limit of the solid constituent and argue that if there are no pores in the solid skeleton, the pore pressure should vanish and hence the effective pressure becomes identical to the confining pressure. Based on this observation, they conclude that,

$B=1-\frac{K}{K_{\mathrm{s}}}$,

where $K$ is the solid skeleton bulk modulus and $K_{\mathrm{s}}$ is the solid grain bulk modulus. The ratio $K / K_{\mathrm{s}}$ equals 1 when the porosity (ratio between the pore volume and the total volume) becomes zero and/or the bulk and solid skeleton bulk modulus are identical. The definition of the effective stress in (1) allows one to separate the constitutive model of the solid skeleton from the pore fluid counterpart and thus simplify the formulation. Considering the limiting case where inertial terms are neglected, the balance of linear momentum under the quasi-static condition thus takes the form,

$\nabla^{\boldsymbol{x}} \cdot\left(\boldsymbol{\sigma}^{\prime}-B p \boldsymbol{I}\right)+\boldsymbol{\gamma}=\mathbf{0}$,

$\boldsymbol{\gamma}=\boldsymbol{\gamma}^{s}+\boldsymbol{\gamma}^{f}=\left(1-\phi^{f}\right) \rho^{s} \boldsymbol{g}+\phi^{f} \rho^{f} \boldsymbol{g}$,

where $\boldsymbol{g}$ is the acceleration due to gravity, $\phi^{f}$ is the porosity (i.e. the ratio between pore and total volume), and $\rho^{s}$ and $\rho^{f}$ are the intrinsic densities of the solid grain and pore fluid (i.e. mass of the constituent divided by the volume occupied by the corresponding constituent). Meanwhile, the balance of angular momentum simply requires the symmetry of the effective stress, $\sigma_{i j}^{\prime}=\sigma_{j i}^{\prime}$.

In porous media, the pore pressure may alter the storage capacity of the pores, while volumetric deformation of the skeleton may change the pore geometry and induce or limit seepage [41-43, 45]. These interactions are taken into account in the balance of mass equation, which takes the following form,

$-B \nabla^{x} \cdot \dot{\boldsymbol{u}}-\frac{1}{M} \dot{p}+\nabla^{x} \cdot \frac{1}{\mu} \boldsymbol{k}\left(\nabla^{x} p+\gamma^{f}\right)=0$,

where $\mu$ is the viscosity of the pore fluid, and $\boldsymbol{k}$ is the intrinsic permeability of the porous system. The scalar parameter $M$ is the Biot modulus referred to in Biot [8] and Coussy [14] and takes the following form,

$M=\frac{K_{\mathrm{s}} K_{\mathrm{f}}}{K_{\mathrm{f}}\left(B-\phi^{f}\right)+K_{\mathrm{s}} \phi^{f}}$, where $K_{\mathrm{s}}$ and $K_{\mathrm{f}}$ are the bulk moduli of the solid grain and the pore fluid constituents.

\subsection{Weak form of the poromechanics problem}

The weak form of the poromechanics problem is obtained by coupling Eqs. (3) and (5) and applying the principle of virtual work. Consider a body $\mathcal{B}$ consisting of material points $\boldsymbol{x} \in R^{n_{\mathrm{dim}}}$ where $n_{\mathrm{dim}}$ is the number of the spatial dimensions. The boundary of $\mathcal{B}$ is denoted by $\partial \mathcal{B}$, which is the direct sum of the Dirichlet and Von Neumann boundaries for displacement and pore pressure,

$\partial \mathcal{B}=\overline{\partial \mathcal{B}_{\boldsymbol{u}} \cup \partial \mathcal{B}_{\boldsymbol{t}}}=\overline{\partial \mathcal{B}_{p} \cup \partial \mathcal{B}_{q}}$,

$\emptyset=\partial \mathcal{B}_{\boldsymbol{u}} \cap \partial \mathcal{B}_{\boldsymbol{t}}=\partial \mathcal{B}_{p} \cap \partial \mathcal{B}_{q}$

where $\partial \mathcal{B}_{u}$ is the solid displacement boundary, $\partial \mathcal{B}_{t}$ is the solid traction boundary, $\partial \mathcal{B}_{p}$ is the pore pressure boundary, and $\partial \mathcal{B}_{q}$ is the pore fluid flux boundary. The boundary conditions are prescribed as,

$\boldsymbol{u}=\overline{\boldsymbol{u}}$ on $\partial \mathcal{B}_{\boldsymbol{u}}$,

$\boldsymbol{n} \cdot \boldsymbol{\sigma}=\overline{\boldsymbol{t}}$ on $\partial \mathcal{B}_{\boldsymbol{t}}$

$p=\bar{p}$ on $\partial \mathcal{B}_{p}$,

$\boldsymbol{- n} \cdot \boldsymbol{q}=\bar{q}$ on $\partial \mathcal{B}_{\boldsymbol{q}}$,

where $\overline{\boldsymbol{u}}$ and $\overline{\boldsymbol{t}}$ are the prescribed skeleton displacement and traction, $\bar{p}$ and $\bar{q}$ are the prescribed pore pressure and the fluid flux. $\boldsymbol{n}$ is the unit normal vector to the boundaries. In addition, we consider the trial space for the weak form which reads,

$V_{\boldsymbol{u}}=\left\{\boldsymbol{u}: \mathcal{B} \rightarrow \mathbb{R}^{3}\left|\boldsymbol{u} \in\left[H^{1}(\mathcal{B})\right]^{3}, \boldsymbol{u}\right|_{\partial_{B_{u}}}=\overline{\boldsymbol{u}}\right\}$,

$V_{p}=\left\{p: \mathcal{B} \rightarrow \mathbb{R}\left|p \in H^{1}(\mathcal{B}), p\right|_{\partial B_{p}}=\bar{p}\right\}$

where $H^{1}$ denotes the Sobolev space of degree one. The admissible variations of displacement $\boldsymbol{\eta}$ and pore pressure $\psi$ therefore read,

$V_{\boldsymbol{\eta}}=\left\{\boldsymbol{\eta}: \mathcal{B} \rightarrow \mathbb{R}^{3}\left|\boldsymbol{\eta} \in\left[H^{1}(\mathcal{B})\right]^{3}, \boldsymbol{\eta}\right|_{\partial B_{\boldsymbol{\eta}}}=\mathbf{0}\right\}$,

$V_{\psi}=\left\{\psi: \mathcal{B} \rightarrow \mathbb{R}\left|\psi \in H^{1}(\mathcal{B}), \psi\right|_{\partial B_{p}}=0\right\}$.

For brevity, the spatial argument $x \in \mathcal{B}$ is not explicitly written. Moreover, the time derivative of function $A$ is simply denoted as $\dot{A}$. The weighted-residual statement of the balance of mass and linear momentum reads: find $\boldsymbol{u} \in$ $V_{\boldsymbol{u}}$ and $p \in V_{p}$ such that for all $\boldsymbol{\eta} \in V_{\boldsymbol{\eta}}$ and $\psi \in V_{\psi}$,

$G(\boldsymbol{u}, p, \boldsymbol{\eta})=H(\boldsymbol{u}, p, \psi)=0$,

where $G: V_{\boldsymbol{u}} \times V_{p} \times V_{\boldsymbol{\eta}} \rightarrow \mathbb{R}$ and $H: V_{\boldsymbol{u}} \times V_{p} \times V_{\psi} \rightarrow \mathbb{R}$ are defined as, 


$$
\begin{aligned}
G(\boldsymbol{u}, p, \boldsymbol{\eta})= & \int_{\mathcal{B}}\left\{\nabla^{\boldsymbol{x}} \cdot \boldsymbol{\eta}:\left(\boldsymbol{\sigma}^{\prime}-B p \boldsymbol{I}\right)-\boldsymbol{\eta} \cdot \boldsymbol{\gamma}\right\} \mathrm{d} V \\
& -\int_{\partial \mathcal{B}_{t}} \boldsymbol{\eta} \cdot \overline{\boldsymbol{t}} \mathrm{d} \Gamma \\
H(\boldsymbol{u}, p, \psi)= & \int_{\mathcal{B}}\left\{\psi B \nabla^{\boldsymbol{x}} \cdot \dot{\boldsymbol{u}}+\psi \frac{1}{M} \dot{p}\right\} \mathrm{d} V \\
& -\int_{\mathcal{B}} \psi \nabla^{\boldsymbol{x}} \cdot \frac{1}{\mu} \boldsymbol{k}\left(\nabla^{\boldsymbol{x}} p+\gamma^{f}\right) \mathrm{d} V \\
& -\int_{\partial B_{q}} \psi \bar{q} \mathrm{~d} \Gamma .
\end{aligned}
$$

\subsection{Assumed strain for volumetric locking}

Standard displacement-based finite element formulations may exhibit a tendency to lock in the nearly incompressible regime. This overly stiff response can be remedied by introducing additional degrees of freedom to decouple the volumetric and deviatoric responses [53]. Nevertheless, introducing additional degrees of freedom may slow down the computation and cause further complications related to the satisfaction of the inf-sup condition. As an alternative, locking can be eliminated by using the assumed strain method [35, 44, 44, 44, 52]. The key idea of the assumed strain method is to consistently replace the strain obtained from the interpolated displacement field by an incompatible strain field. Since no additional solution fields are introduced, this method is more efficient than the mixed field method. Nevertheless, additional stabilization mechanisms must be introduced into the finite element formulation in order to eliminate spurious modes, such as the hourglass mode discussed in Wriggers [52].

In this work, we introduce an assumed strain formulation which derives directly from the assumed deformation gradient method presented in Sun et al. [44]. This assumed strain formulation is based on the fact that the infinitesimal strain tensor is the linearized strain measure of both the Green-Lagrange strain tensor and the Eulerian strain tensor, i.e.

$\boldsymbol{\epsilon}=\frac{1}{2}\left(\nabla^{\boldsymbol{x}} \cdot \boldsymbol{u}+\left(\nabla^{\boldsymbol{x}} \boldsymbol{u}\right)^{T}\right)=\frac{1}{2}\left(\boldsymbol{F}+\boldsymbol{F}^{T}-2 \boldsymbol{I}\right)$.

Hence, one may define an assumed strain field by replacing the standard deformation gradient with an incompatible, assumed deformation gradient that can overcome the volumetric locking, i.e.

$\overline{\boldsymbol{\epsilon}}=\frac{1}{2}\left(\overline{\boldsymbol{F}}+\overline{\boldsymbol{F}}^{T}-2 \boldsymbol{I}\right)$

For instance, one may employ the logarithm-based volumetric approximated deformation gradient by Moran et al. [25] which reads,
$\overline{\boldsymbol{F}}\left(\boldsymbol{\xi}_{i}\right)=\left(\frac{\exp \left[\sum_{j=1}^{n_{I}} \log \left(J\left(\overline{\boldsymbol{\xi}}_{j}\right) \bar{N}_{j}\left(\boldsymbol{\xi}_{i}\right)\right]\right.}{J\left(\boldsymbol{\xi}_{i}\right)}\right)^{1 / 3} \boldsymbol{F}\left(\boldsymbol{\xi}_{i}\right)$

where $\boldsymbol{\xi}_{i}$ are the integration points, $J=\operatorname{det} \boldsymbol{F}$, and $\bar{N}_{j}$ is the prescribed function which generates the approximate Jacobian of $\boldsymbol{F} . n_{I}$ is the number of sampling points for $J . \overline{\boldsymbol{\xi}}_{j}\left(j=1,2, \ldots, n_{I}\right)$ denotes the prescribed local coordinates of the sampling points for $J$ such that $\bar{N}_{j}\left(\bar{\xi}_{i}\right)=\delta_{i j}$ de Souza Neto et al. [15].

Here we adopt the refinement in Sun et al. [44], of which a combined standard/F-bar element approach is used to ensure stabilization, as seen in the following equation,

$\widetilde{\boldsymbol{F}}\left(\boldsymbol{\xi}_{i}\right)=\widetilde{J}\left(\boldsymbol{\xi}_{i}\right)^{1 / 3} \boldsymbol{J}\left(\boldsymbol{\xi}_{i}\right)^{-1 / 3} \boldsymbol{F}\left(\boldsymbol{\xi}_{i}\right)$

where

$\widetilde{J}\left(\xi_{i}\right)=\exp \left((1-\alpha)\left(\sum_{j=1}^{n_{I}} \log \left(J\left(\bar{\xi}_{j}\right) \bar{N}_{j}\left(\xi_{i}\right)\right)+\alpha \log J\left(\xi_{i}\right)\right)\right.$,

where $\alpha \in[0,1]$. The combined formulation may reduce to the standard or F-bar formulation by adjusting $\alpha$. Furthermore, it can be easily shown that (23) is identical to the mid-point assumed deformation gradient formulation in de Souza Neto et al. [15] if $\alpha=0$ and the volume averaging of $\log J(X)$ is computed via one-point quadrature at the centroid of the element. In all the simulations presented in this paper, we found that setting $\alpha=0.05$ appeared to eliminate the zero energy modes.

\subsection{Projection stabilization for equal-order discretization}

Near the undrained limit, the pore fluid trapped inside the pore space may cause the skeleton to lose its compressibility as a consequence of the incompressibility of the pore fluid. In that case, the mixed finite element formulation is unstable unless (1) the trial and the solution space of displacement and pore pressure both satisfy the inf-sup condition $[3,4,5,12]$ or (2) a stabilization procedure is applied [9, 44, 51].

In either case, the objective is to make sure that the kernel of the solution space of the pore pressure field does not contain any non-trivial spurious modes,

$$
\begin{aligned}
\operatorname{ker}\left(\nabla^{h}\right) & =\left\{p_{h} \in V_{p}^{h} \int_{\mathcal{B}} p_{h} \nabla^{\boldsymbol{x}} \cdot \boldsymbol{u}_{h} d V=0 \forall \boldsymbol{u} \in V_{\boldsymbol{u}}{ }^{h}\right\} \\
& =\{\mathbf{0}\},
\end{aligned}
$$

where $V_{p}^{h} \subset V_{p}$ and $V_{\boldsymbol{u}}^{h} \subset V_{\boldsymbol{u}}$ are the finite dimensional spaces of the displacement and pore pressure, and $\nabla^{h}$ is the discrete gradient operator defined in $V_{\boldsymbol{u}}{ }^{h}$. If (25) does not hold, then a non-trivial spurious pressure mode may exist and thus there could be multiple admissible solutions for Eqs. $(18,19)$. 
The presence of spurious pore pressure modes can be interpreted as a signal that the pore pressure space is comparatively too rich for the displacement space. Therefore, the strategy employed to eliminate the spurious zero energy modes includes restricting the pore pressure space properly. Similar problems also arise when equal-order velocity-pressure bases are used in the solution of the incompressible Navier-Stokes problem. For instance, [9] uses the weaker inf-sup bound to derive stabilization procedures for both the $Q 1 P 0$ and $Q 1 P 1$ finite elements. This stabilization procedure is adopted in White and Borja [51] to solve the poromechanics problem with incompressible solid and fluid constituents.

Recall that a stable and accurate approximation to the saddle point for the pair of $V_{p}^{h}$ and $V_{\boldsymbol{u}}{ }^{h}$ satisfies the discrete inf-sup condition, i.e.

$\sup _{\boldsymbol{u}_{h} \in V_{\boldsymbol{u}}^{h}, \boldsymbol{u}_{h} \neq \mathbf{0}} \frac{\int_{\mathcal{B}} p_{h} \nabla^{x} \cdot \boldsymbol{u}_{h} d V}{\left\|\boldsymbol{u}_{h}\right\|_{1}} \geq \gamma\left\|p^{h}\right\|_{0}, \forall p^{h} \in V_{p}^{h}$,

where $\gamma$ is a positive constant independent of the mesh size. As pointed out in Bochev et al. [9], while an equal-order pair $V_{p}^{h}$ and $V_{u}{ }^{h}$ does not satisfy (26), it does satisfy a weaker bound

$\sup _{\boldsymbol{u}_{h} \in V_{\boldsymbol{u}^{h}, \boldsymbol{u}_{h} \neq \mathbf{0}}} \frac{\int_{\mathcal{B}} p_{h} \nabla^{x} \boldsymbol{u}_{h} d V}{\left\|\boldsymbol{u}_{h}\right\|_{1}} \geq \gamma_{1}\left\|p^{h}\right\|_{0}-\gamma_{2} h\left\|\nabla^{x} p^{h}\right\|_{0}, \forall p^{h} \in V_{p}^{h}$,

where $\gamma_{1}$ and $\gamma_{2}$ are both positive constants independent of the mesh size.

For poromechanics problems with nonlinear constitutive laws, the inf-sup condition cannot be directly applied. However, one can apply the inf-sup condition to the tangent of the linearized governing equations. In this case, a stabilization term is added to (19) in order to penalize the deficiency in the displacement/pore-pressure approximation pair. This penalty term reads

$R_{\text {stab }}=\int_{\mathcal{B}^{e}} \tau(\psi-\Pi(\psi))(\dot{p}-\Pi(\dot{p})) \mathrm{d} V$,

where $\tau$ is the stabilization parameter. $\Pi$ is a projection operator that maps the pore pressure onto a element-wise constant function,

$\Pi(p)=\frac{\sum_{j=1}^{n_{l}} p\left(\boldsymbol{\xi}_{j}\right) w\left(\boldsymbol{\xi}_{j}\right)}{\sum_{i=1}^{n_{I}} w\left(\boldsymbol{\xi}_{i}\right)} ; p\left(\boldsymbol{\xi}_{j}\right)=\sum_{a=1}^{n_{\text {node }}} N_{a}\left(\boldsymbol{\xi}_{j}\right) p_{a}$

where $\Pi(\cdot)$ is a constant within a single finite element consisting of $n_{I}$ integration points and $w\left(\xi_{j}\right)$ is the associated weight corresponding to the $j$ th integration points for numerical integrations. $N_{a}\left(\xi_{j}\right)$ and $p_{a}$ are the interpolation function and the nodal pore pressure corresponding to the node $a$ where $n_{\text {node }}$ denotes the number of nodes per element.

\section{Three-invariant cap plasticity model}

Cap models are typically used in the modeling of complicated behavior of porous geomaterials, taking into account pressure-sensitive yielding, differences in strength in triaxial compression and extension, the Bauschinger effect, dependence on porosity, and so on. They are capable of capturing shear localization in low porosity geomaterials. Stefanov et al. [39] has shown that a carefully calibrated cap model may also reproduce compactive shear and pure compactive deformation bands in high-porosity geomaterials.

In this section, the formulation and numerical implementation of a three-invariant, isotropic and kinematic hardening cap plasticity model are briefly described. The model is composed of a pressure-dependent shear yield surface and a hardening compaction cap as shown in Fig. 1. Notice that in Fig. 1, the sign convention is compression positive while in the rest of the paper, compression is treated as negative. For more details and motivation of the model, the reader is referred to $[18,19]$. The numerical integration of the cap model is performed using a refined explicit algorithm with a normal correction as detailed later in this section.

\subsection{Yield functions and plastic potentials}

Before introducing the yield and plastic potential functions, a deviatoric backstress tensor $\boldsymbol{\alpha}$ is presented to capture the Bauschinger effect for cyclic loading, such that the relative stress tensor can be defined as $\xi=\boldsymbol{\sigma}-\boldsymbol{\alpha}$. The yield function $f$ and plastic potential function $g$ are then both written in terms of stress invariants of the relative stress tensor, i.e. $J_{2}^{\xi}$ and $J_{3}^{\xi}$, where the superscript $\xi$ shows the stress invariant is written in term of relative stress tensor. The stress invariant $I_{1}$ is unchanged since the backstress tensor $\boldsymbol{\alpha}$ is deviatoric in nature. The yield function and plastic potential function are given as,

$f\left(I_{1}, J_{2}^{\xi}, J_{3}^{\xi}, \boldsymbol{\alpha}, \kappa\right)=(\Gamma(\beta))^{2} J_{2}^{\xi}-F_{c}\left(F_{f}-N\right)^{2}=0$,

$g\left(I_{1}, J_{2}^{\xi}, J_{3}^{\xi}, \boldsymbol{\alpha}, \kappa\right)=(\Gamma(\beta))^{2} J_{2}^{\xi}-F_{c}^{g}\left(F_{f}^{g}-N\right)^{2}$,

where $F_{f}$ is an exponential shear failure function, and $F_{f}^{g}$ is the corresponding plastic potential surface given as,

$F_{f}\left(I_{1}\right)=A-C \exp \left(D I_{1}\right)-\theta I_{1}$,

$F_{f}^{g}\left(I_{1}\right)=A-C \exp \left(L I_{1}\right)-\phi I_{1}$.

The shear failure surface $F_{f}$ (as shown in Fig. 1b) captures the pressure-dependence of the shear strength of the material. $N$ is a material parameter that represents the offset of the yield function from the shear failure surface. $A, C, D$ and $\theta$ are material parameters that are fit to 


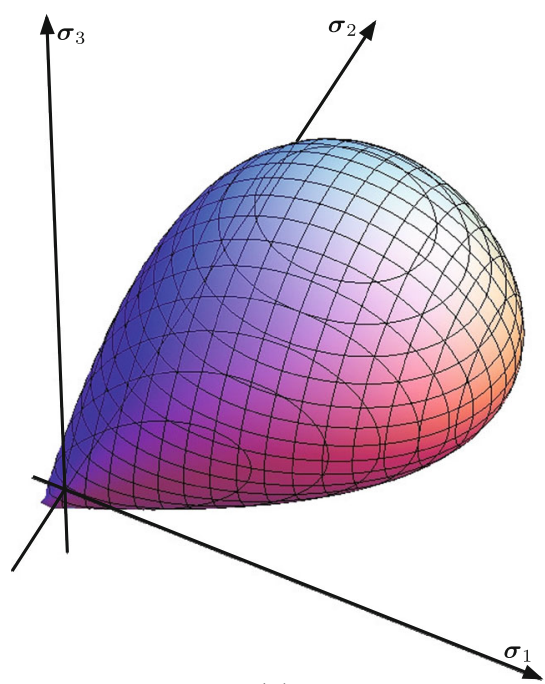

(a)

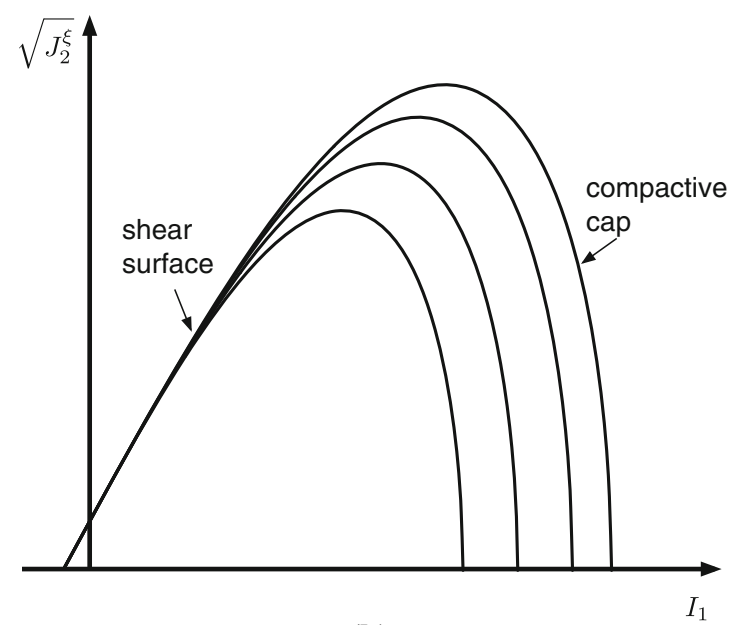

(b)

Fig. 1 Cap plasticity model yield surface: a three-dimensional representation in principal stress space; b two-dimensional representation in meridional stress space; deviatoric stress $\sqrt{J_{2}}$ versus mean stress $I_{1}$. Sign conventions in this figure: compression positive and tension negative

experimental peak stress for various confining pressures. $L$ and $\phi$ are determined from experimental measurements of volumetric plastic deformation. $\Gamma(\beta)$ is a function of the Lode angle, which takes into account the differences in strength in triaxial compression and extension tests, as typically seen in geomaterials. The Lode angle $\beta$ is given as

$$
\beta=-\frac{1}{3} \sin ^{-1}\left(\frac{3 \sqrt{3} J_{3}^{\xi}}{2\left(J_{2}^{\xi}\right)^{3 / 2}}\right),
$$

and $\Gamma(\beta)$ is introduced as

$$
\begin{aligned}
& \Gamma(\beta)=\frac{1}{2}\left(1+\sin (3 \beta)+\frac{1}{\psi}(1-\sin (3 \beta))\right) \\
& =\frac{1}{2}\left(1-\frac{3 \sqrt{3} J_{3}^{\xi}}{2\left(J_{2}^{\xi}\right)^{3 / 2}}+\frac{1}{\psi}\left(1+\frac{3 \sqrt{3} J_{3}^{\xi}}{2\left(J_{2}^{\xi}\right)^{3 / 2}}\right)\right),
\end{aligned}
$$

where $\psi$ is the ratio of triaxial extension strength to compression strength. $F_{c}$ provides a smooth elliptical cap to the yield function and is given by

$F_{c}\left(I_{1}, \kappa\right)=1-H\left(\kappa-I_{1}\right)\left(\frac{I_{1}-\kappa}{X(\kappa)-\kappa}\right)^{2}$,

where $H\left(\kappa-I_{1}\right)$ is the Heaviside function. The effect of the function $F_{c}$ is that at some value of mean stress, $\kappa$, the yield surface begins to deviate from the initial shear yield surface, until a point $X$ is reached, where there is no shear strength. The function $X(\kappa)$ is the intersection of the cap surface with the $I_{1}$ axis in the $\sqrt{J_{2}}$ versus $I_{1}$ plane and is given by

$X(\kappa)=\kappa-R F_{f}(\kappa)$, where $R$ is a material parameter governing the aspect ratio of the cap surface. The corresponding functions for the plastic potential $g$ are written as,

$F_{c}^{g}\left(I_{1}, \kappa\right)=1-H\left(\kappa-I_{1}\right)\left(\frac{I_{1}-\kappa}{X^{g}(\kappa)-\kappa}\right)^{2}$,

$X^{g}(\kappa)=\kappa-Q F_{f}(\kappa)$,

where $Q$ is a material parameter analogous to $R$.

\subsection{Evolution laws for isotropic/kinematic hardening parameters}

There are two internal variables involved in the cap plasticity model: the cap hardening parameter $\kappa$ for isotropic hardening, and the deviatoric back stress $\boldsymbol{\alpha}$ for kinematic hardening. The evolution of $\kappa$ is related to the volumetric plastic strain, $\epsilon_{v}^{p}$, while the evolution of $\boldsymbol{\alpha}$ is related to the deviatoric plastic strain, $\boldsymbol{e}^{p}$. The evolution of $\boldsymbol{\alpha}$ is given by the following expression

$\dot{\alpha}=\dot{\gamma} \boldsymbol{h}^{\alpha}(\boldsymbol{\alpha})$,

$\boldsymbol{h}^{\alpha}=c^{\alpha} G^{\alpha}(\boldsymbol{\alpha}) \operatorname{dev}\left(\frac{\partial g}{\partial \boldsymbol{\sigma}}\right)$,

where $\dot{\gamma}$ is the consistency parameter, and $c^{\alpha}$ is a material parameter that controls the rate of hardening. $G^{\alpha}(\boldsymbol{\alpha})$ is a function which limits the growth of the back stress as it approaches the failure surface and can be written as

$G^{\alpha}(\boldsymbol{\alpha})=1-\frac{\sqrt{J_{2}^{\alpha}}}{N}, \quad J_{2}^{\alpha}=\frac{1}{2} \boldsymbol{\alpha}: \boldsymbol{\alpha}$.

The evolution of $\kappa$, related to isotropic hardening, is given as 
$\dot{\kappa}=\dot{\gamma} h^{\kappa}(\kappa)$,

$h^{\kappa}=\frac{\operatorname{tr}(\partial \mathrm{g} / \partial \boldsymbol{\sigma})}{\partial \epsilon_{v}^{p} / \partial \kappa}$.

The following form of volumetric plastic strain is used, $\epsilon_{v}^{p}=W\left(\exp \left[D_{1}\left(X(\kappa)-X_{0}\right)-D_{2}\left(X(\kappa)-X_{0}\right)^{2}\right]-1\right)$.

In the above, $W, D_{1}$ and $D_{2}$ are material parameters, $X_{0}=X\left(\kappa_{0}\right)$ is the initial cap position with $\kappa_{0}$ being the initial value of the cap parameter.

\subsection{Flow rule and generalized Hooke's law}

To complete the formulation for the cap plasticity model at small strains, an additive decomposition of the strain rate tensor into elastic and plastic parts is introduced,

$\dot{\boldsymbol{\epsilon}}=\dot{\boldsymbol{\epsilon}}^{e}+\dot{\boldsymbol{\epsilon}}^{p}$.

We assume the existence of a plastic potential function (e.g. (31)) that governs the direction of plastic flow via

$\dot{\boldsymbol{\epsilon}}^{p}=\dot{\gamma} \frac{\partial g}{\partial \boldsymbol{\sigma}}$

where $\dot{\gamma}$ is the plastic multiplier or consistency parameter. For associative flow, $g=f$. For linear isotropic elasticity, the generalized Hooke's law is written as

$\dot{\boldsymbol{\sigma}}=\boldsymbol{c}^{e}: \dot{\boldsymbol{\epsilon}}^{e}=\boldsymbol{c}^{e}:\left(\dot{\boldsymbol{\epsilon}}-\dot{\boldsymbol{\epsilon}}^{p}\right)$,

where $c^{e}$ is the fourth-order isotropic elasticity tensor

$\boldsymbol{c}^{e}=\lambda \boldsymbol{I} \otimes \boldsymbol{I}+2 \mu \mathbb{I} ; \mathbb{I}=1 / 2\left(\delta_{i k} \delta_{j l}+\delta_{i l} \delta_{j k}\right) \boldsymbol{e}_{i} \otimes \boldsymbol{e}_{j} \otimes \boldsymbol{e}_{k} \otimes \boldsymbol{e}_{l}$,

and $\boldsymbol{I}$ is the second-order identity tensor, $\mathbb{I}$ is the symmetric part of the fourth-rank identity tensor, $\delta_{i j}$ is the Kronecker delta, $\boldsymbol{e}_{i}$ is the Cartesian basis vector, $\lambda$ and $\mu$ are Lamé constants.

\subsection{Numerical integration of the constitutive model}

Given the values of stress and the internal variables at time $t_{n}$, and the strain increment $\Delta \epsilon$, the goal of the numerical integration is to find the stress and internal variable values at time $t_{n+1}$. This is accomplished using the evolution Eqs. $(41,44,48,49)$, while at the same time satisfying the yield condition (30). In this work, we employ a refined explicit integration algorithm. This refined explicit scheme is advantageous over the implicit counterpart, in the sense that it is easier to implement and does not require linearizing and solving a system of nonlinear equations. In the cap plasticity model, the number of unknowns can be as large as 13 (6 for stress components, 5 for kinematic hardening parameter, 1 for isotropic hardening and 1 for plastic multiplier), but this number can be reduced to 7 using a modified spectral decomposition method as shown in Foster et al. [19]. Alternatively, a semi-implicit algorithm proposed by $\mathrm{Tu}$ et al. [48] for integrating nonsmooth elasto-plasticity models may be applied which keeps the internal variables constant and delays the update until local convergence is achieved, effectively reducing the number of unknowns in the system of nonlinear equations.

The explicit integration scheme adopted in this work includes an algorithm to prevent the stress and hardening parameters from drifting away from the yield surface. To that end, a normal correction algorithm [38] is employed. The integration scheme consists of an elastic trial state followed by a plastic-corrector as described in Algorithm 1. Once the plastic update is obtained, the yield surface is evaluated again to determine if a drift has occurred. The extent of the drifting depends on the accuracy of the integration scheme and the nonlinearity of the constitutive models. The stress correction algorithm to prevent such drifting is detailed in Algorithm 2. This algorithm uses a correction normal to the yield surface to correct the drifted stress. Notice that the expression for $\tilde{\chi}$ in Algorithm 2 is due to the assumption of holding hardening parameters unchanged while using normal correction.

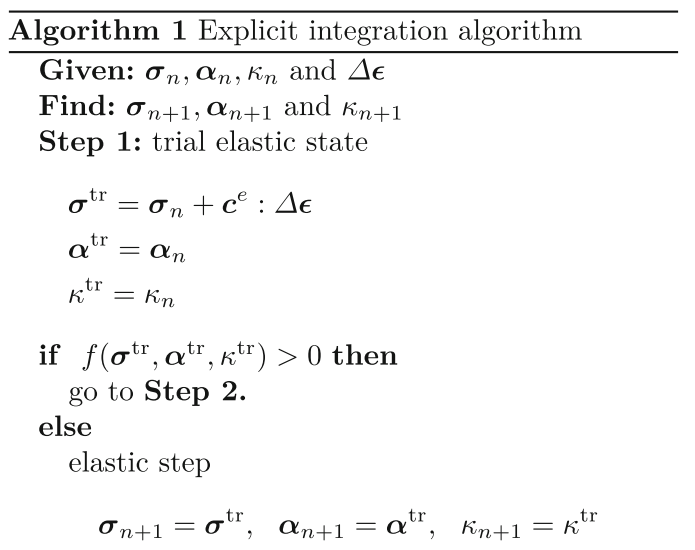

end if

Step 2: plastic correction

$$
\begin{aligned}
& \boldsymbol{\sigma}_{n+1}=\boldsymbol{\sigma}^{\mathrm{tr}}-\Delta \gamma \frac{\partial g_{n}}{\partial \boldsymbol{\sigma}} \\
& \boldsymbol{\alpha}_{n+1}=\boldsymbol{\alpha}_{n}+\Delta \gamma \boldsymbol{h}_{\alpha n} \\
& \kappa_{n+1}=\kappa_{n}+\Delta \gamma h_{\kappa n} \\
& g_{n}=g\left(\boldsymbol{\sigma}_{n}, \boldsymbol{\alpha}_{n}, \kappa_{n}\right)
\end{aligned}
$$

where

$$
\begin{aligned}
& \Delta \gamma=\frac{1}{\chi}\left(\frac{\partial f_{n}}{\partial \boldsymbol{\sigma}}: \boldsymbol{c}^{e}: \boldsymbol{\Delta} \epsilon\right) \\
& f_{n}=f\left(\boldsymbol{\sigma}_{n}, \boldsymbol{\alpha}_{n}, \kappa_{n}\right) \\
& \chi=\frac{\partial f_{n}}{\partial \boldsymbol{\sigma}}: \boldsymbol{c}^{e}: \frac{\partial g_{n}}{\partial \boldsymbol{\sigma}}-\frac{\partial f_{n}}{\partial \boldsymbol{\alpha}}: \boldsymbol{h}_{\alpha n}-\frac{\partial f_{n}}{\partial \kappa} h_{\kappa n} \\
& \text { if }\left|f\left(\boldsymbol{\sigma}_{n+1}, \boldsymbol{\alpha}_{n+1}, \kappa_{n+1}\right)\right|>\text { tolerance then } \\
& \text { go to stress correction (Algorithm 2) } \\
& \text { else } \\
& \text { exit with current states }\left(\boldsymbol{\sigma}_{n+1}, \boldsymbol{\alpha}_{n+1}, \kappa_{n+1}\right) \\
& \text { end if }
\end{aligned}
$$




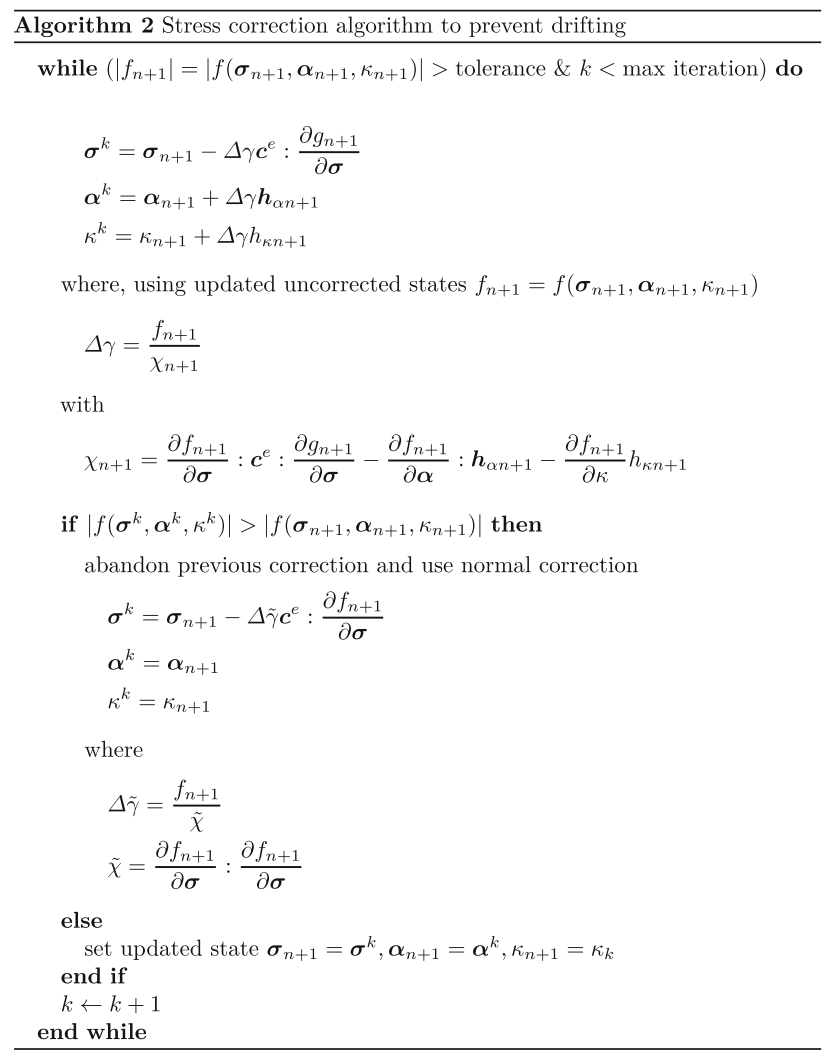

\subsection{Material properties for Salem limestone}

To replicate the effective stress-strain relation of the Salem limestone, we employ two sets of material parameters reported in Fossum and Fredrich [18], Foster et al. [19], Regueiro and Foster [30]. The first set of material parameters, which is listed in Table 1, is for modeling associative plastic behavior of Salem limestone. For comparison, we also employ a second set of material parameters reported in Regueiro and Foster [30] to replicate non-associative plastic behavior. This set of material parameters is listed in Table 2 .

In both cases, the hydro-mechanical coupling and the hydraulic properties are captured via the additional material parameters listed in Table 3. All numerical simulations reported in Sect. 5 are conducted with the two sets of material parameters reported in Tables 1 and 2, along with the hydro-mechanical coupling and hydraulic parameters listed in Table 3.

\section{Stress-induced permeability changes}

Adequately modeling the hydro-mechanical response of porous media requires constitutive models for both the solid skeleton (e.g. the cap plasticity model), and the pore fluid (e.g. Darcy's law). While significant effort has been spent on developing sophisticated constitutive models to handle the solid skeleton response in porous continua [19,
Table 1 Material parameters for the cap model fitted to Salem limestone data

\begin{tabular}{|c|c|c|c|}
\hline Parameter & Description & Value & Unit \\
\hline E & Young's modulus & 22,547 & $\mathrm{MPa}$ \\
\hline$v$ & Poisson's ratio & 0.2524 & Dimensionless \\
\hline$A$ & $\begin{array}{l}\text { Shear failure } \\
\text { parameter }\end{array}$ & 689.2 & $\mathrm{MPa}$ \\
\hline$C$ & $\begin{array}{l}\text { Shear failure } \\
\text { parameter }\end{array}$ & 675.2 & $\mathrm{MPa}$ \\
\hline$D, L$ & $\begin{array}{c}\text { Shear failure } \\
\text { parameter }\end{array}$ & $\begin{array}{c}3.94 \times 10^{-4} \\
3.94 \times 10^{-4}\end{array}$ & $1 / \mathrm{MPa}$ \\
\hline$\theta, \phi$ & $\begin{array}{l}\text { Shear failure } \\
\text { parameter }\end{array}$ & $0.0,0.0$ & $\operatorname{Rad}$ \\
\hline$R$ & Cap parameter & 28.0 & Dimensionless \\
\hline$\kappa_{0}$ & Initial cap position & -8.05 & $\mathrm{MPa}$ \\
\hline$W$ & $\begin{array}{l}\text { Parameter for } \epsilon_{v}^{p} \\
\text { function }\end{array}$ & 0.08 & Dimensionless \\
\hline$D_{1}$ & $\begin{array}{l}\text { Parameter for } \epsilon_{v}^{p} \\
\text { function }\end{array}$ & $1.47 \times 10^{-3}$ & $1 / \mathrm{MPa}$ \\
\hline$D_{2}$ & $\begin{array}{l}\text { Parameter for } \epsilon_{v}^{p} \\
\text { function }\end{array}$ & 0.0 & $1 / \mathrm{MPa}^{2}$ \\
\hline$c^{\alpha}$ & Bulk modulus & $1 e 5$ & $\mathrm{MPa}$ \\
\hline$\psi$ & $\begin{array}{l}\text { Triaxial extension to } \\
\text { compression strength } \\
\text { ratio }\end{array}$ & 1.0 & Dimensionless \\
\hline$N$ & $\begin{array}{l}\text { Initial yield surface } \\
\text { offset }\end{array}$ & 6.0 & $\mathrm{MPa}$ \\
\hline
\end{tabular}

19, 21, 22, 22, 24, 41], permeability, which governs the solid-fluid interaction inside the porous media, is often assumed to be constant or related to porosity via simple models [6]. Recent researches, such as [20, 34], Yang and Aplin [54], have incorporated other factors, such as pore throat distributions, pore shapes, effective stress and damages into empirical or semi-empirical formulas to improve the accuracy of permeability predictions.

To quantitatively assess how porosity evolution leads to changes in hydraulic response, we perform a regression analysis and obtain the best fitted coefficient, $k_{\mathrm{o}}$, for the Kozeny-Carman equation based on the Salem limestone $\log$ data from Stevenson [40], i.e.

$k=k_{o} \frac{\left(\phi^{f}\right)^{3}}{\left(1-\phi^{f}\right)^{2}}$.

Meanwhile, volumetric deformation of the skeleton may lead to shrinkage or enlargement of the pore space. This effect is modeled via the Biot's coefficient and Biot's modulus (cf. [14]),

$\dot{\phi}^{f}=B\left(\dot{\epsilon}_{i i}^{e}+\dot{\epsilon}_{i i}^{p}\right)+\left(\frac{1}{M}-\frac{\phi^{f}}{K_{\mathrm{f}}}\right) \dot{p}$.

Note that if the stress state lies on the cap surface, then part of the porosity change is irreversible. Figure 2 compares 
Table 2 Material parameters for the non-associative cap plasticity model. Non-associativity is introduced through $L$ and $\phi$

\begin{tabular}{|c|c|c|c|}
\hline Parameter & Description & Value & Unit \\
\hline E & Young's modulus & 22,547 & $\mathrm{MPa}$ \\
\hline$v$ & Poisson's ratio & 0.2524 & Dimensionless \\
\hline$A$ & Shear failure parameter & 689.2 & $\mathrm{MPa}$ \\
\hline C & Shear failure parameter & 675.2 & $\mathrm{MPa}$ \\
\hline$D, L$ & Shear failure parameter & $\begin{array}{r}3.94 \times 10^{-4} \\
3.5 \times 10^{-4}\end{array}$ & $1 / \mathrm{MPa}$ \\
\hline$\theta, \phi$ & shear failure parameter & $0.1,0.085$ & $\operatorname{Rad}$ \\
\hline$R$ & Cap parameter & 28.0 & Dimensionless \\
\hline$\kappa_{0}$ & Initial cap position & -8.05 & $\mathrm{MPa}$ \\
\hline$W$ & Parameter for $\epsilon_{v}^{p}$ function & 0.08 & Dimensionless \\
\hline$D_{1}$ & Parameter for $\epsilon_{v}^{p}$ function & $1.47 \times 10^{-3}$ & $1 / \mathrm{MPa}$ \\
\hline$D_{2}$ & Parameter for $\epsilon_{v}^{p}$ function & 0.0 & $1 / \mathrm{MPa}^{2}$ \\
\hline$c^{\alpha}$ & Bulk modulus & $1 e 5$ & $\mathrm{MPa}$ \\
\hline$\psi$ & $\begin{array}{l}\text { Triaxial extension to } \\
\text { compression strength } \\
\text { ratio }\end{array}$ & 1.0 & Dimensionless \\
\hline$N$ & Initial yield surface offset & 6.0 & $\mathrm{MPa}$ \\
\hline
\end{tabular}

Table 3 Additional material parameters used in the poromechanics formulation

\begin{tabular}{llll}
\hline Parameter & Description & Value & Unit \\
\hline$K_{\mathrm{s}}$ & $\begin{array}{c}\text { Bulk modulus of the } \\
\text { solid constituent }\end{array}$ & $5 \times 10^{4}$ & $\mathrm{MPa}$ \\
$K_{\mathrm{f}}$ & $\begin{array}{c}\text { Bulk modulus of the } \\
\text { pore fluid }\end{array}$ & $2.2 \times 10^{3}$ & $\mathrm{MPa}$ \\
$k_{0} / \mu$ & $\begin{array}{c}\text { Kozeny-Carman fitting } \\
\text { coefficient }\end{array}$ & $2.176 \times 10^{-9}$ & $\mathrm{~m}^{2} /(\mathrm{Pa} \mathrm{s})$ \\
$\phi^{f}$ & \begin{tabular}{c} 
Porosity \\
\hline
\end{tabular} & 0.1 & Dimensionless \\
\hline
\end{tabular}

the best fit Kozeny-Carman equation (red line) with the log data from Stevenson [40]. We found that the best fit $k_{\mathrm{o}}=17.76$ Darcy, while $R^{2}=0.585$.

We assume that the isothermal condition holds. The pore fluid is at $20{ }^{\circ} \mathrm{C}$ where the viscosity of the pore fluid is $10^{-3} \mathrm{~Pa} \cdot$ second. When the porosity equals to $0.1, \mathrm{k} / \mu=$ $2.69 \times 10^{-12} \mathrm{~m} /(\mathrm{Pa} \mathrm{s})$, the approximate hydraulic conductivity is $2.63 \times 10^{-8} \mathrm{~m} / \mathrm{s}$.

\section{Numerical examples}

In this section, we present a one-dimensional confined compression loading, an unconfined drained shear loading, and two punch loading simulations. Our objective is to verify the implementation and examine how factors such as loading rate, drainage of pore fluid, and pore collapse affect the stability of the fully saturated porous rock. In addition, we also use the examples to assess the numerical

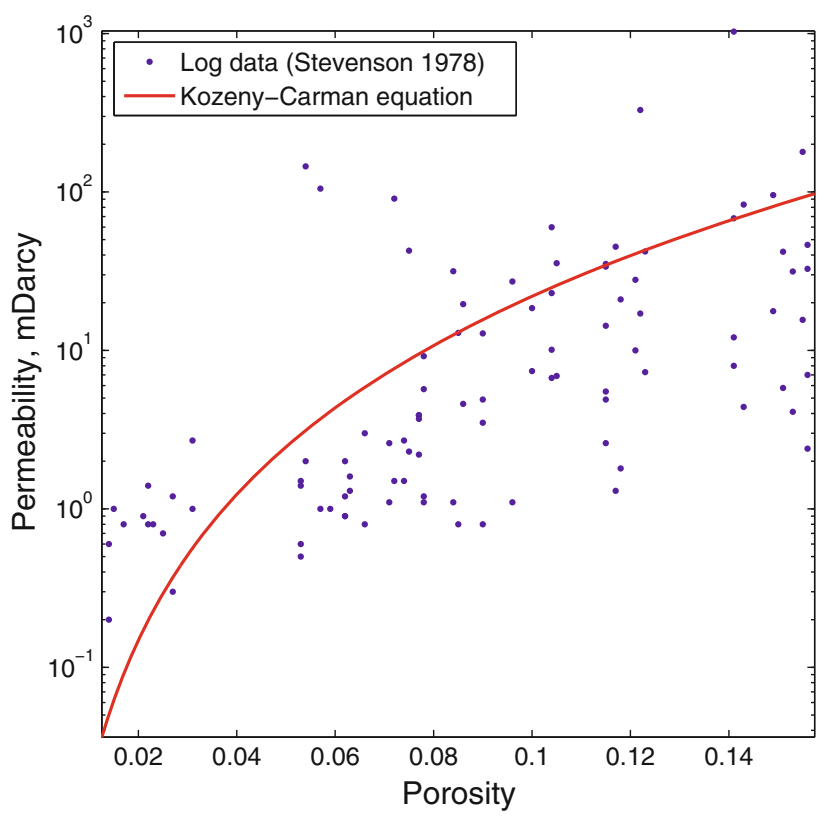

Fig. 2 Kozeny-Carman equation compared against log data from Stevenson [40]

performance of the stabilized mixed finite element with assumed strain formulation. Gravity effects are neglected in the numerical examples.

\subsection{One-dimensional compression loading}

Previously, the one-dimensional loading test has been used to verify the implementation for the finite strain poroelasticity problem in the context of the stabilized finite element formulation in Sun et al. [44]. Here we re-use this onedimensional confined test for two purposes: (1) to verify the finite element implementation with the assumed strain formulation and (2) to examine the hydro-mechanical coupling effect in a simple loading path.

For the sake of completeness, we briefly outline the boundary value problem for uniaxial confined compression. First, a fully saturated stress free numerical specimen is placed in a cylindrical hydrated chamber such that no pore fluid exchange occurs at the bottom and the side boundaries. As a result, the pore fluid flux is zero at those locations. The height, $h$, of the cylinder is $30 \mathrm{~m}$, and the radius of the cylinder is 30 $\mathrm{m}$. Horizontal displacement is constrained to simulate onedimensional compression condition. A ramp-and-hold downward displacement is applied on the top surface where water is free to flow in and out of the articular layer, i.e.

$u(z=h, t)=\left\{\begin{array}{cc}-\gamma t & \text { if } t_{o} \geq t \geq 0 \\ -\gamma t_{o} & \text { if } t>t_{0}\end{array}\right.$,

where $\gamma$ is the loading rate. The analytical solution of this problem is available if both constituents are assumed to be 


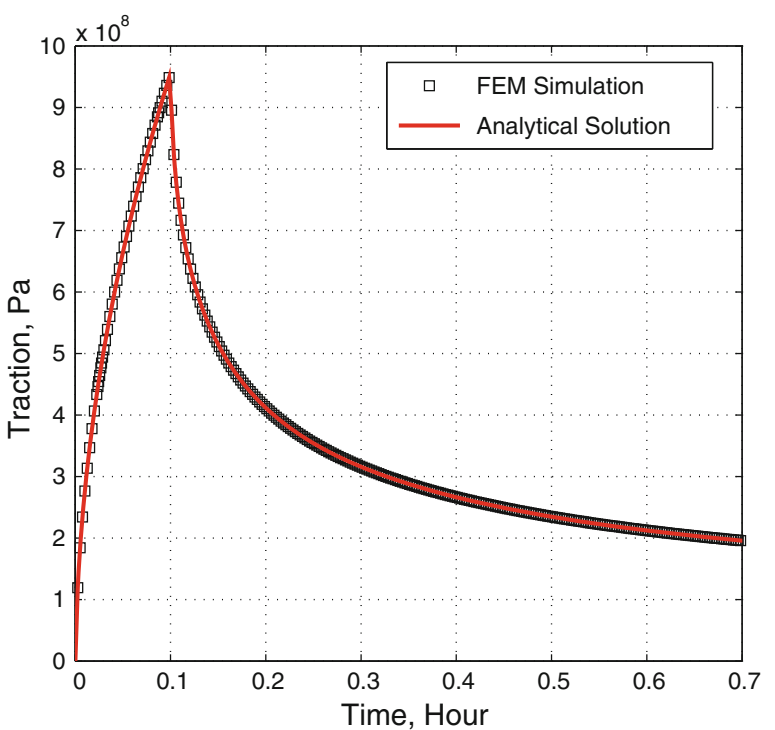

Fig. 3 One-dimensional compression loading on a fully saturated porous medium with incompressible constituents. Traction responses obtained via the stabilized finite element method and analytical solution at the top of the fully saturated specimen with incompressible constituents $\left(\gamma=1.5 \mathrm{~m} / \mathrm{h}\right.$ and $\left.t_{o}=0.1 \mathrm{~h}\right)$

incompressible and the permeability tensor is isotropic. In this case the traction on the top surface reads,

$$
\begin{aligned}
\left.t_{3}(t)\right|_{z=h}= & \frac{\gamma H_{A}}{h}\left[t H\left(t_{o}-t\right)+t_{o} H\left(t-t_{o}\right)\right] \\
& +\frac{2 \gamma h}{\kappa} \sum_{n=1}^{\infty} \frac{1}{n^{2} \pi^{2}}\left[H\left(t_{o}-t\right)\left(1-e^{-a_{n} t}\right)\right. \\
& \left.+H\left(t-t_{o}\right)\left(e^{-a_{n}\left(t-t_{o}\right)}-e^{-a_{n} t}\right)\right],
\end{aligned}
$$

where $H$ is the Heaviside step function. $H_{A}=\lambda+2 G$ is the sum of the Lamé's first parameter and two times the shear modulus, $a_{n}=n^{2} \pi^{2} \kappa H_{A} / h^{2}$ and $\kappa=k / v$ is the intrinsic permeability $k$ divided by the viscosity $v$.

Due to the axisymmetry, only a quarter of the domain is modeled. To verify the implementation and ensure that the assumed strain methodology does not introduce errors, we conducted finite element simulations of an elastic porous layer with the same elastic parameter in Table 1, but fixed the porosity, and set $K_{\mathrm{s}}$ and $K_{\mathrm{f}}$ equal to $1,000 \mathrm{GPa}$, approximating both solid and fluid constituents as incompressible. Figure 3 shows the finite element solution and the analytical solution obtained via Eq. (54) for $\gamma=0.15$ $\mathrm{m} / \mathrm{h}$ and $t_{o}=0.1 \mathrm{~h}$. Notice that the analytical and numerical solutions are in excellent agreement. This indicates that the assumed strain formulation is able to capture the analytical solution when both the solid grain and pore fluid are incompressible.

Next, we employ the elasto-plastic cap model and set both the bulk modulus of the solid grain, $K_{\mathrm{s}}$, and pore fluid, $K_{\mathrm{f}}$, equal to the assigned values in Table 3 . As a result of

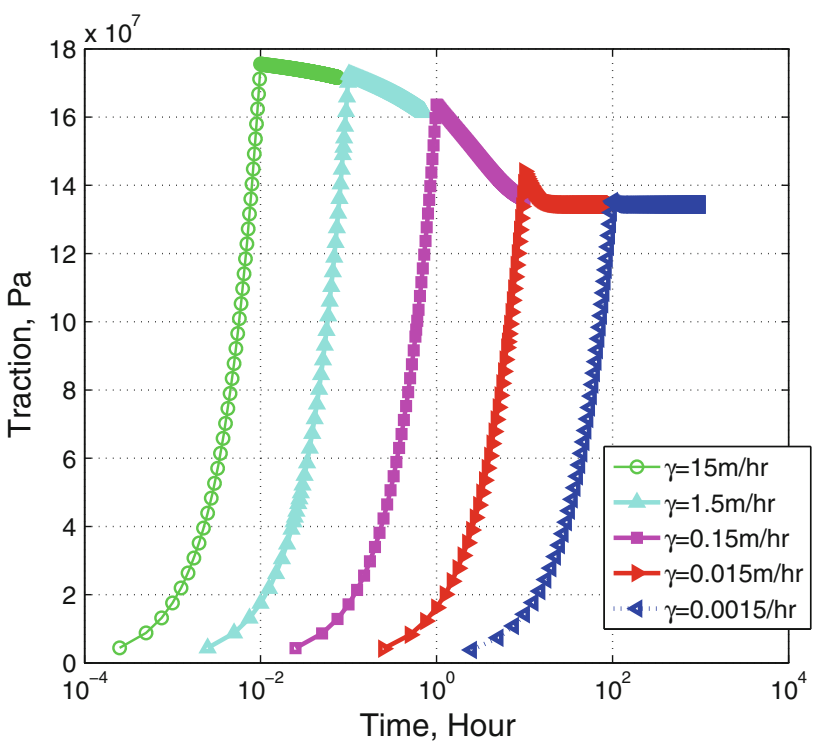

Fig. 4 One-dimensional compression loading with associative cap plasticity. Traction responses for different loading rates

the compressibility of the constituents, the Biot's coefficient $B=0.7$ and the Biot's modulus $M=18 \mathrm{GPa}$. Five loading rates are prescribed as $\gamma=0.0015,0.015,0.15,1.5$ and $15 \mathrm{~m} / \mathrm{h}$. Figure 4 compares the finite element solutions obtained at the five different loading rates. Notice that although the prescribed displacement is the same, the maximum traction decreases from 180 to $136 \mathrm{MPa}$, apparently due to the decreased loading rate. Also notice that the plasticity model is time independent, and thus, the rate effect is solely due to the coupling between the solid deformation and pore fluid diffusion. Figure 5a shows the stress path in $\sqrt{J_{2}^{\xi}}$ versus $I_{1}$ plane. Of all five simulations with different rates, plastic response only occurs when $\gamma=0.15,1.5$ and $15 \mathrm{~m} / \mathrm{h}$. In Fig. 5b, the stress paths are plotted in $\sqrt{J_{2}}$ versus $I_{1}$ plane, along with the final failure surface. As expected, the stress paths appear to deviate from the yield surface because the principal directions of the relative stress tensor $\boldsymbol{\xi}$ are changing.

The difference in mechanical responses among different cases is due to the hydro-mechanical coupling effect. At higher loading rates, the interaction occurs near the undrained limit, where the pore fluid acts as a volumetric constraint to the solid skeleton in a time scale smaller than that of the diffusion process. Thus, a higher traction must be applied to achieve the same prescribed displacement, and this higher traction leads to plastic yielding and the development of a plastic zone near the top layer of the confined specimen. By contrast, the mechanical response of the porous layer is purely elastic when the slow loading rates $(\gamma=0.0015,0.015 \mathrm{~m} / \mathrm{h})$ are prescribed. These results indicate that the external work due to the prescribed 


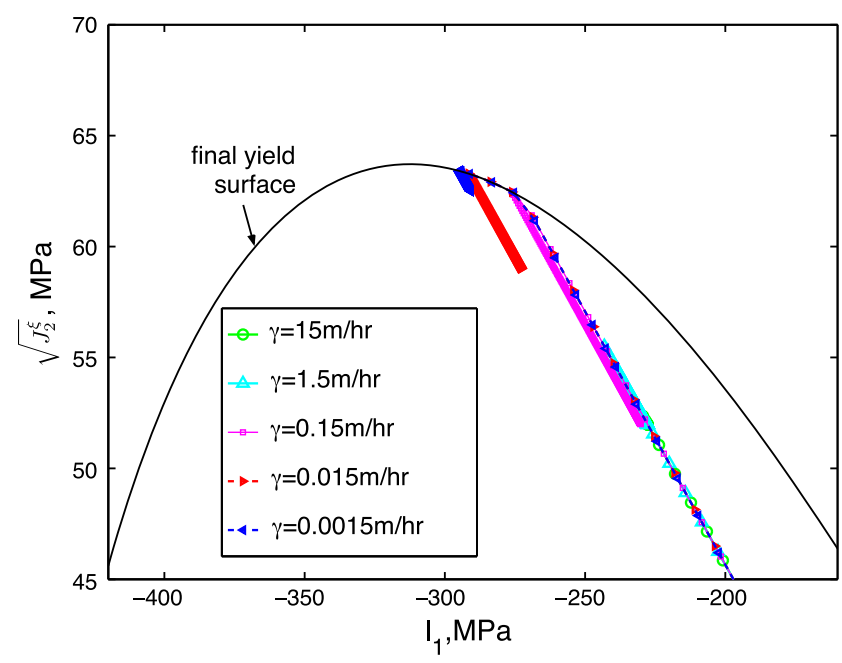

(a)

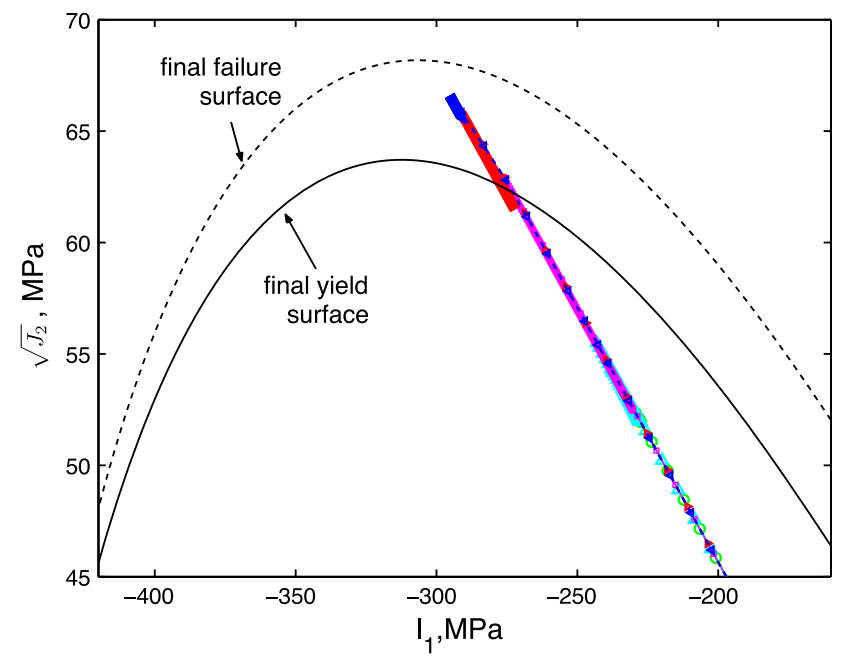

(b)

Fig. 5 One-dimensional compression loading with associative cap plasticity. Stress path of the material point nearest to the top layer loaded at different loading rates: a in meridional stress space $\sqrt{J_{2}^{\xi}}$ versus $I_{1} ; \mathbf{b}$ in meridional stress space $\sqrt{J_{2}}$ versus $I_{1}$

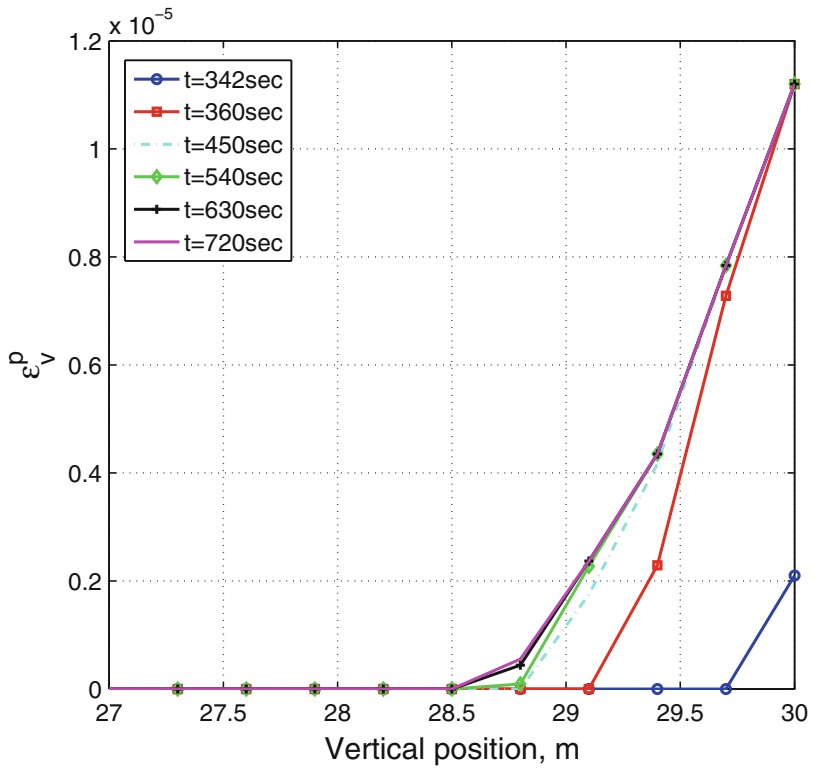

Fig. 6 One-dimensional compression loading with associative cap plasticity. Plastic volumetric strain profile at various time steps with loading rate $\gamma=1.5 \mathrm{~m} / \mathrm{h}$

displacement induces substantial pore fluid diffusion but not plastic work if the loading rate is close to the drained limit.

While the prescribed displacement is held constant, the pore-diffusion process may continue until the fluid reaches steady state. During this period, the plastic zone may propagate from the top layer until the onset of elastic unloading. As shown in Fig. 6, the plastic zone would expand from the top layer to around $1.5 \mathrm{~m}$ from

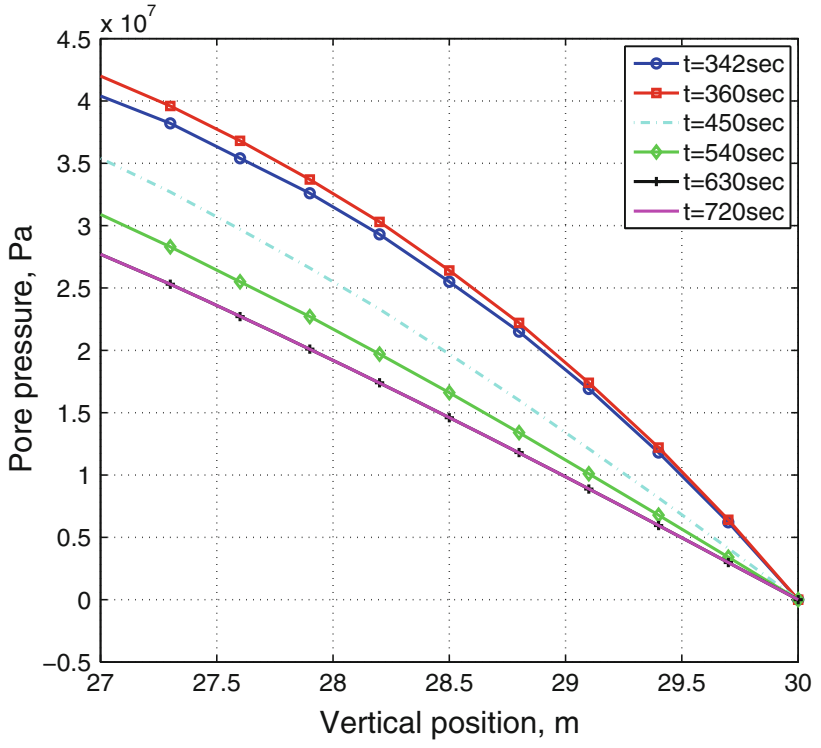

Fig. 7 One-dimensional compression loading with associative cap plasticity. Pore pressure profile at various time steps with loading rate $\gamma=1.5 \mathrm{~m} / \mathrm{h}$

the top surface as pore fluid diffuses. However, when the pore pressure profile becomes linear via the vertical axis, as shown in Fig. 7, steady state is reached and the plastic zone remains the same between $t=630$ and $720 \mathrm{~s}$. Notice that the hydro-mechanical coupling effect showcased in this one-dimensional example could not be properly modeled if the transient diffusion effect was neglected. As a result, oversimplifying these problems as fully drained or undrained may lead to significant errors. 


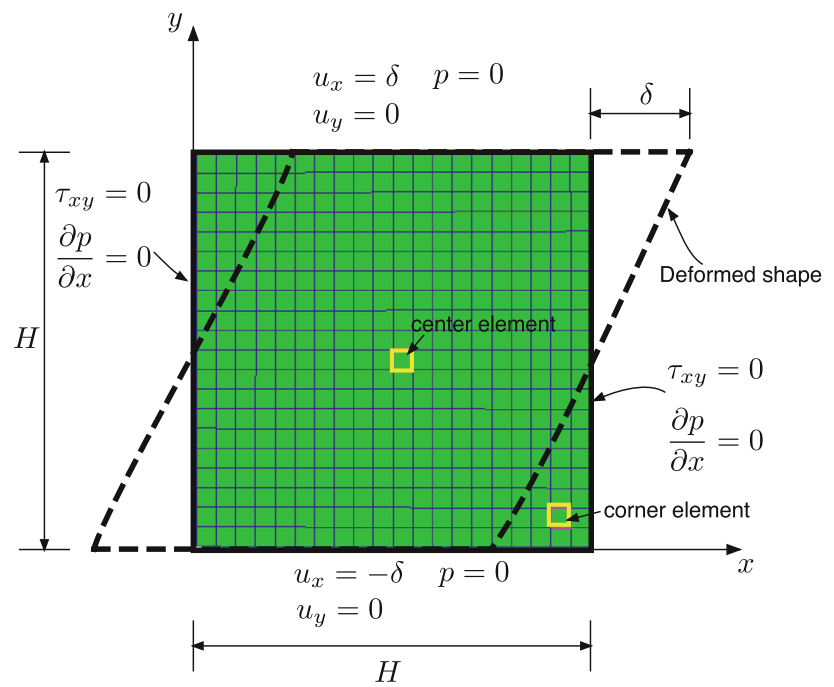

Fig. 8 Boundary conditions of the unconfined drained shear loading. $H=10 \mathrm{~m}$ in the numerical simulations. Structured mesh (fine) shown in the undeformed configuration, where highlighted elements will be selected to report stress paths

\subsection{Unconfined drained shear loading}

The purpose of introducing the unconfined drained shear loading simulation is (1) to analyze how local pore fluid diffusion may affect the formation of a shear band and (2) to provide a numerical assessment of the rate effect induced by the seepage on the mechanical responses. Previously, similar studies on the effects of boundary conditions and local drainage have been conducted in Prevost and Høeg [29], Wang et al. [50]. In this numerical example, we use the non-associative cap-plasticity model and the corresponding material parameters listed in Tables 2 and 3. The boundary conditions are illustrated in Fig. 8. The excess pore pressure at the top and bottom of the specimen is prescribed to be zero to simulate surfaces that permit drainage, while zeroflux boundary conditions are applied on the lateral sides. Gravity is neglected. The vertical displacement of the top and bottom of the specimen is fixed during the shear phase [50], while horizontal displacement is accumulated at a constant rate for a fixed period of time.
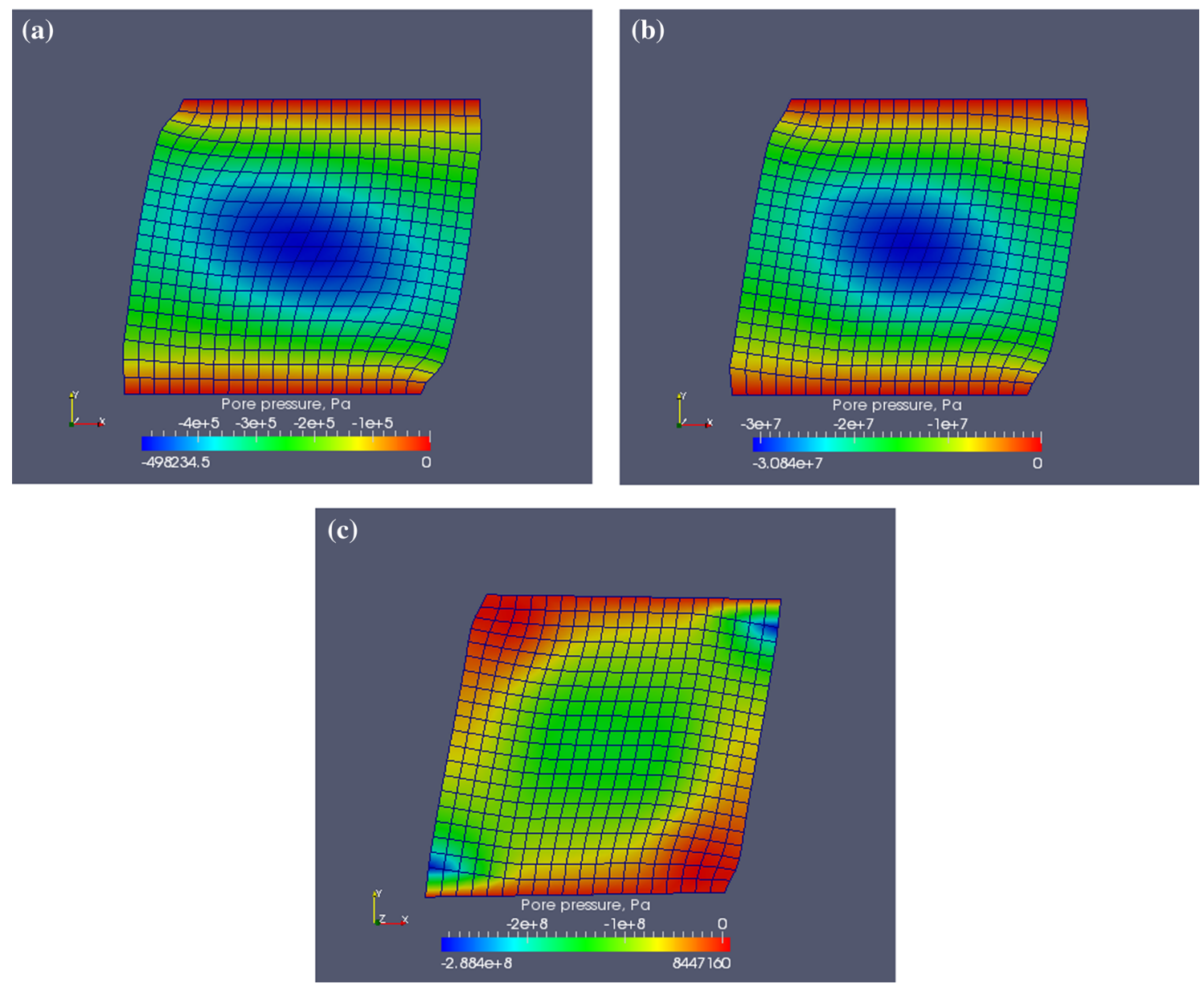

Fig. 9 Unconfined drained shear loading with non-associative cap plasticity. Excess pore pressure built-up at $10 \%$ shear strain under different loading rates. a $10^{-6} \mathrm{~m} / \mathrm{s}$ loading rate, $\mathbf{b} 10^{-4} \mathrm{~m} / \mathrm{s}$ loading rate, $\mathbf{c} 10^{-2} \mathrm{~m} / \mathrm{s}$ loading rate 

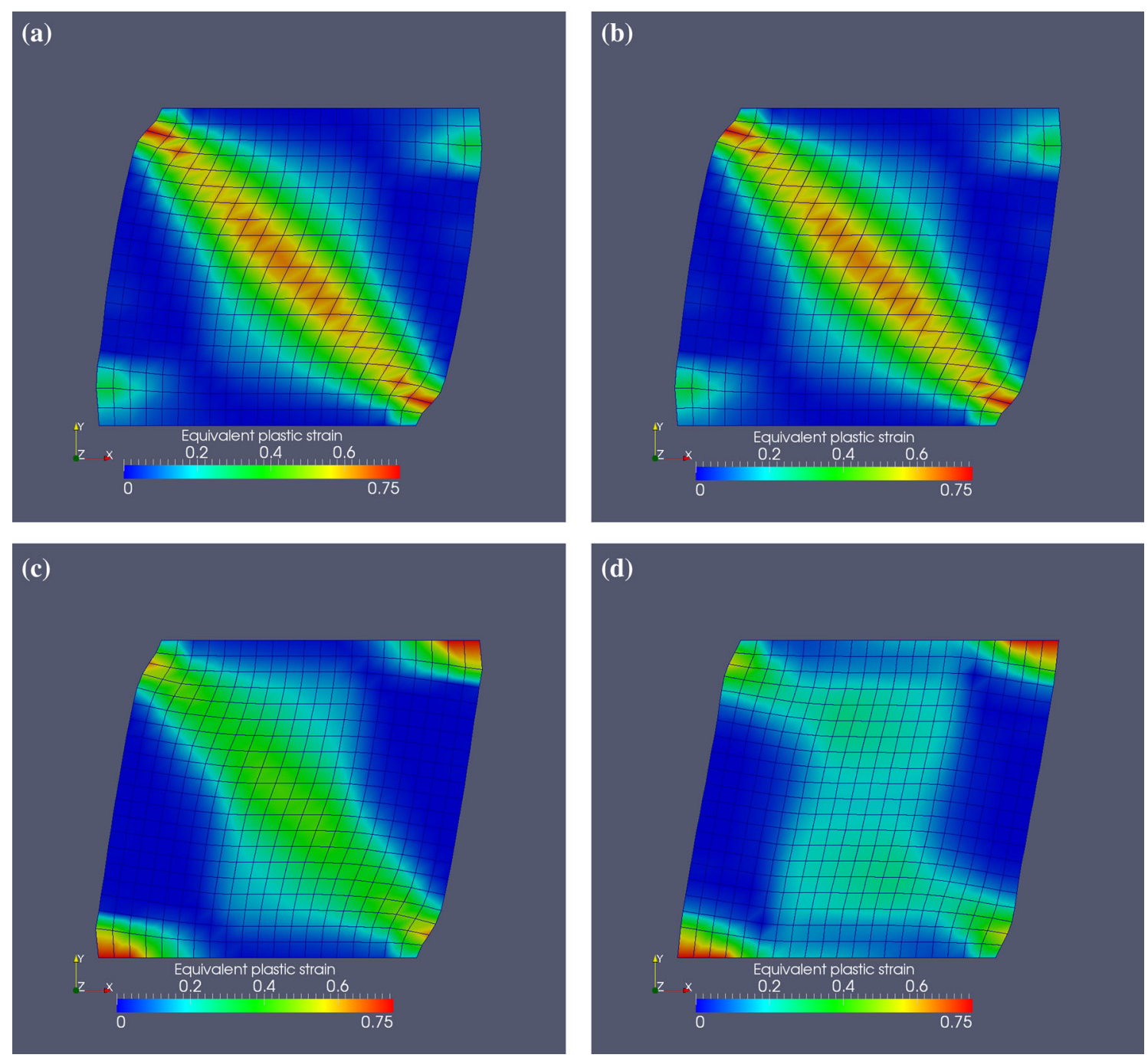

Fig. 10 Unconfined drained shear loading with non-associative cap plasticity. Equivalent plastic strain contours at $10 \%$ shear strain for the dry simulation and poromechanical coupling simulations under different loading rates. a Dry case, $\mathbf{b} 10^{-6} \mathrm{~m} / \mathrm{s}$ loading rate, $\mathbf{c} 10^{-4} \mathrm{~m} / \mathrm{s}$ loading rate, d $10^{-2} \mathrm{~m} / \mathrm{s}$ loading rate

\subsubsection{Rate effect}

In this section, we assess the importance of rate effects on local excess pore pressure build-up and analyze whether the non-uniformity of excess pore pressure causes significant effects on the mechanical response. For comparison, we prescribed the horizontal displacement of the top and bottom of the specimens at three different rates$10^{-6}, 10^{-4}, 10^{-2} \mathrm{~m} / \mathrm{s}$. This difference in prescribed loading rates leads to various amounts of shear-induced diffusion and pore pressure build-up. Figure 9 demonstrates the amount of excess pore pressure generated when the prescribed shear strain reaches $10 \%$. Note that the simulations are all globally drained. In an idealized situation where local seepage is neglected, all simulations are expected to yield the same result. However, these simulations indicate that the local pore fluid diffusion may introduce discrepancies in mechanical response if the ratio between loading rate and hydraulic conductivity is sufficiently high. In addition, the local pore pressure build-up during the shear phase influences the effective stress path and elastoplastic responses. Figures 10 and 11 compare the equivalent plastic strain and the volumetric plastic strain developed at various loading rates in fully saturated and dry simulations. For illustration purposes, quantities such as equivalent and volumetric plastic strains at integration points are projected onto the nodes via a global $L_{2}$ projection [26].

By comparing the plastic responses shown in Figs. 10 and 11 , we observe that the two-way, hydro-mechanical coupling effect is more substantial when the prescribed loading rate is high. For instance, the equivalent plastic strain and volumetric plastic strain profiles of the dry case and the coupled simulation with a $10^{-6} \mathrm{~m} / \mathrm{s}$ loading rate are 

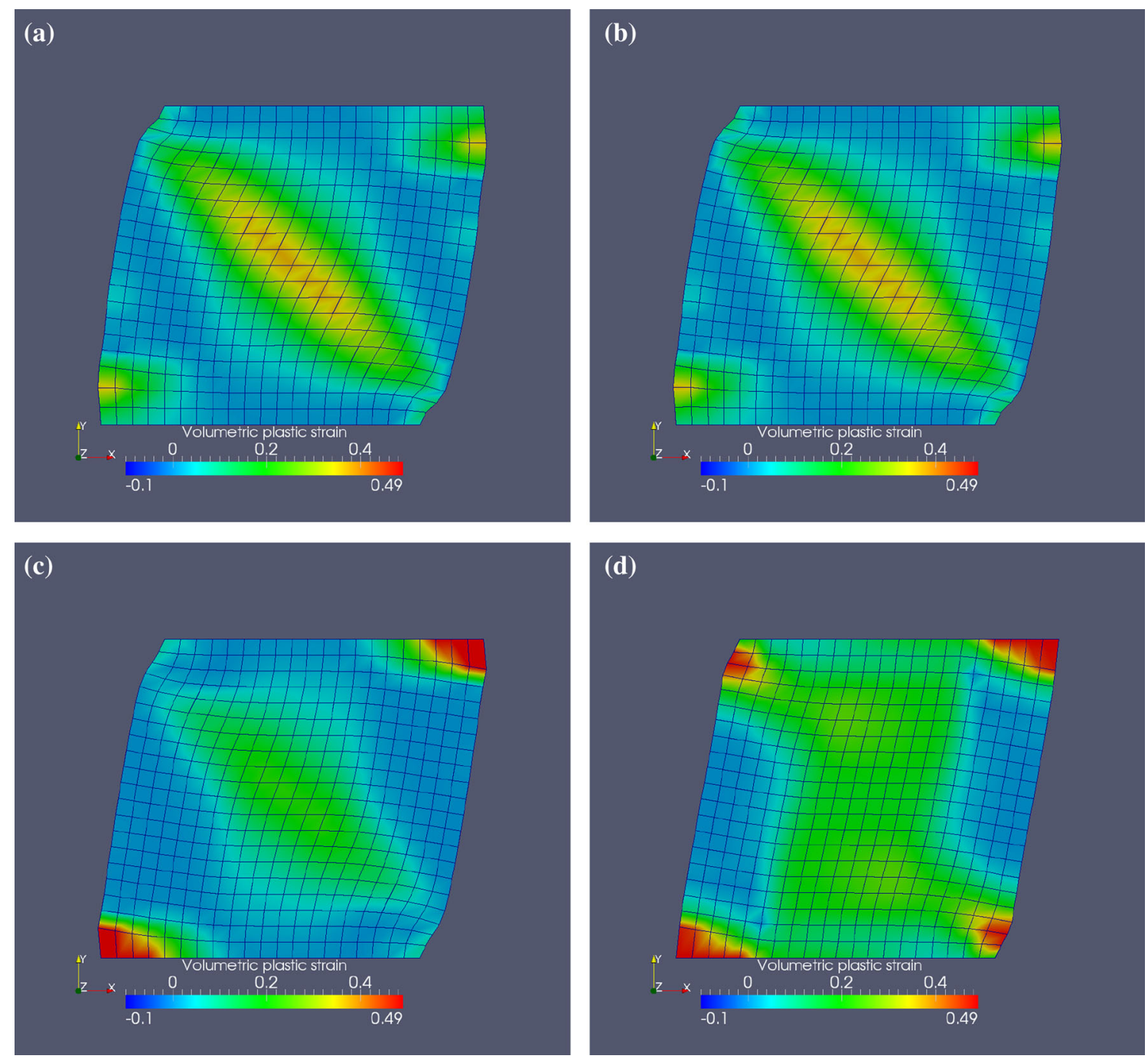

Fig. 11 Unconfined drained shear loading with non-associative cap plasticity. Volumetric plastic strain contours at $10 \%$ shear strain for the dry simulation and poromechanical coupling simulations under different loading rates. a Dry case, $\mathbf{b} 10^{-6} \mathrm{~m} / \mathrm{s}$ loading rate, $\mathbf{c} 10^{-4} \mathrm{~m} / \mathrm{s}$ loading rate, d $10^{-2} \mathrm{~m} / \mathrm{s}$ loading rate

similar to each other, as shown in Figs. 10a, b and 11a, b. While a shear band still forms in the $10^{-4} \mathrm{~m} / \mathrm{s}$ loading case, the specimen exhibits less equivalent plastic strain (from 0.75 to 0.4 ) and volumetric plastic strain (from 0.4 to 0.2 ) in the shear band zone, as shown in Fig. 10c. When loading rate reaches $10^{-2} \mathrm{~m} / \mathrm{s}$, the hydro-mechanical effect is found to be sufficient to prevent the formation of shear band, as shown in Fig. 10d.

In the highest loading rate case, $10^{-2} \mathrm{~m} / \mathrm{s}$, the pore fluid trapped at the middle of the specimen causes a significant pore pressure that prevents the formation of the shear band. More importantly, it clearly shows that fully saturated porous media under drained conditions may behave very differently than the dry solid skeleton if the loading rate is high. In other words, the results of the simulation indicate that constant volume simulations can be used for specimens under drained conditions only when the specimen is loaded very slowly (e.g. $10^{-6} \mathrm{~m} / \mathrm{s}$ ) and gravity is neglected.

As demonstrated by the non-uniform plastic response in Figs. 10 and 11, local stress paths at different locations differ significantly. Figure 12 shows the relative stress paths obtained from the center and the right bottom corner of the specimen at various loading rates (locations of selected elements highlighted in Fig. 8). At both locations, the effective stress response of the slowest loading rate $\left(10^{-6} \mathrm{~m} / \mathrm{s}\right)$ and the dry case coincide with each other. This similarity in stress path and the small pore pressure shown in Fig. 9 indicate that the hydro-mechanical coupling effect is weak at a slow loading rate. However, when the loading rate increases, more negative pore pressure is accumulated 


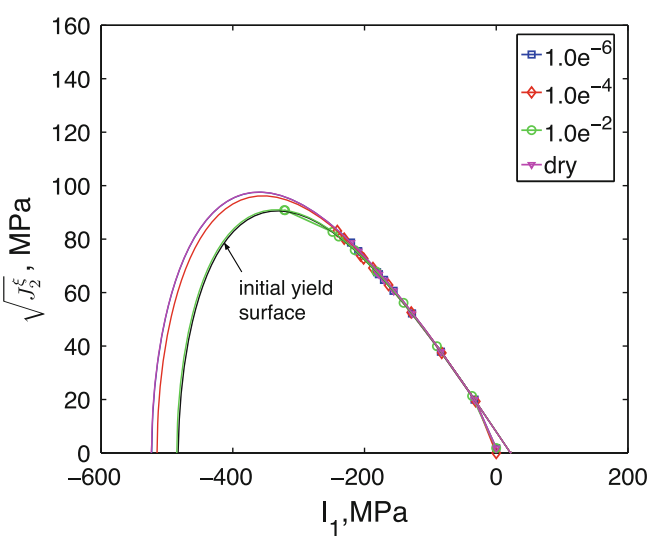

(a)

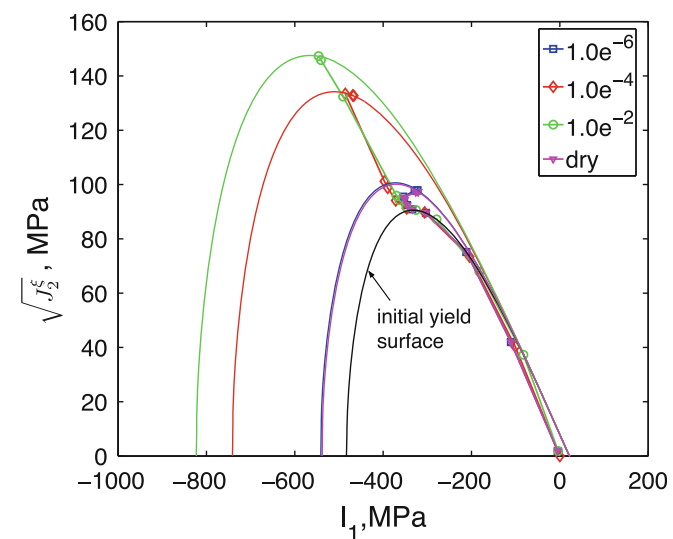

(b)

Fig. 12 Unconfined drained shear loading with non-associative cap plasticity. Relative effective stress path in meridional stress space $\sqrt{J_{2}^{\xi}}$ versus $I_{1}$ for structured fine mesh (locations of the selected elements shown in Fig. 8). The solid lines are the final yield surfaces, colored to match different loading rates as marked in the figure. Notice that for the center element, the yield surface for loading rate $10^{-2} \mathrm{~m} / \mathrm{s}$ is very close to the original yield surface. a Center element, $\mathbf{b}$ corner element

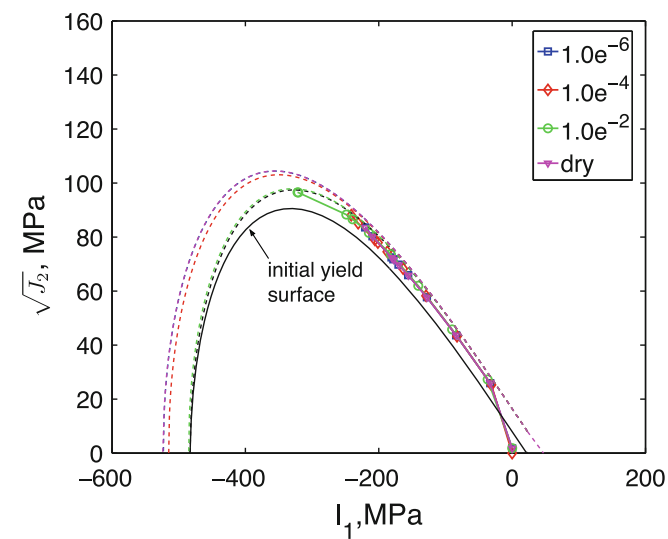

(a)

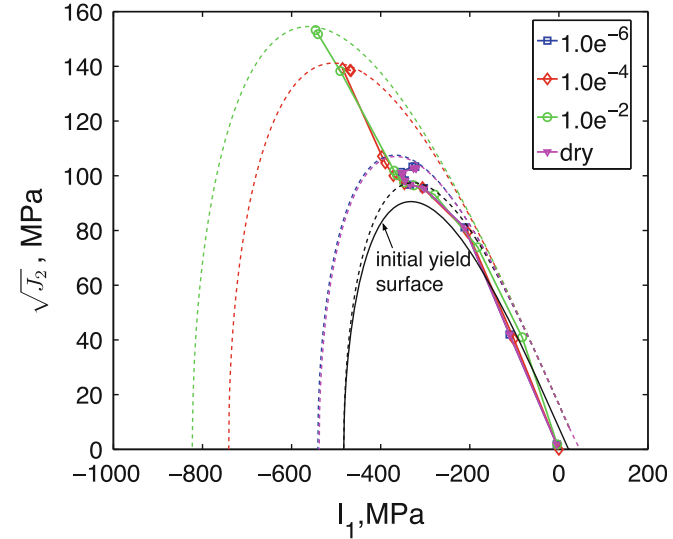

(b)

Fig. 13 Unconfined drained shear loading with non-associative cap plasticity. Effective stress path in meridional stress space $\sqrt{J_{2}}$ versus $I_{1}$ for structured fine mesh (locations of the selected elements shown in Fig. 8). The dashed lines are the final failure surfaces, colored to match different loading rates as marked in the figure. Notice that for the center element, the final failure surface for loading rate $10^{-2} \mathrm{~m} / \mathrm{s}$ is very close to the original failure surface. a Center element, $\mathbf{b}$ corner element

and thus pushes the specimen closer to the undrained limit, which ultimately leads to higher effective hydrostatic and deviatoric stress in the center of the specimen.

\subsubsection{Mesh sensitivity}

The mechanical response of porous media is rate dependent, as long as the flow regime is not at the fully drained or the undrained limit. This rate dependence exists regardless of whether the constitutive law of the solid skeleton is rate sensitive or not, because rate dependence can be introduced through the interaction of the pore fluid and the solid skeleton.

A consequence of this rate dependence is the potential regularization as discussed in Belytschko et al. [7], such that the fully coupled hydro-mechanical field equations are stable and free of mesh pathology if sufficient drainage occurs. One interpretation is that the physical length scale introduced by the pore fluid diffusion is independent of the mesh size. This hypothesis is confirmed in one dimension through a dynamic wave propagation analysis of saturated porous media [1, 55]. Previously, Regueiro and Foster [30] conducted a bifurcation analysis on the three-invariant cap plasticity model incorporated in this study. Their findings indicate that bifurcations may occur when the solid skeleton response is non-associative and rate independent. However, the role of pore fluid diffusion on mesh dependence was not given consideration in Regueiro and Foster [30].

Figure 14 compares the equivalent plastic strain accumulated from two uniform meshes consisting of 100 and 

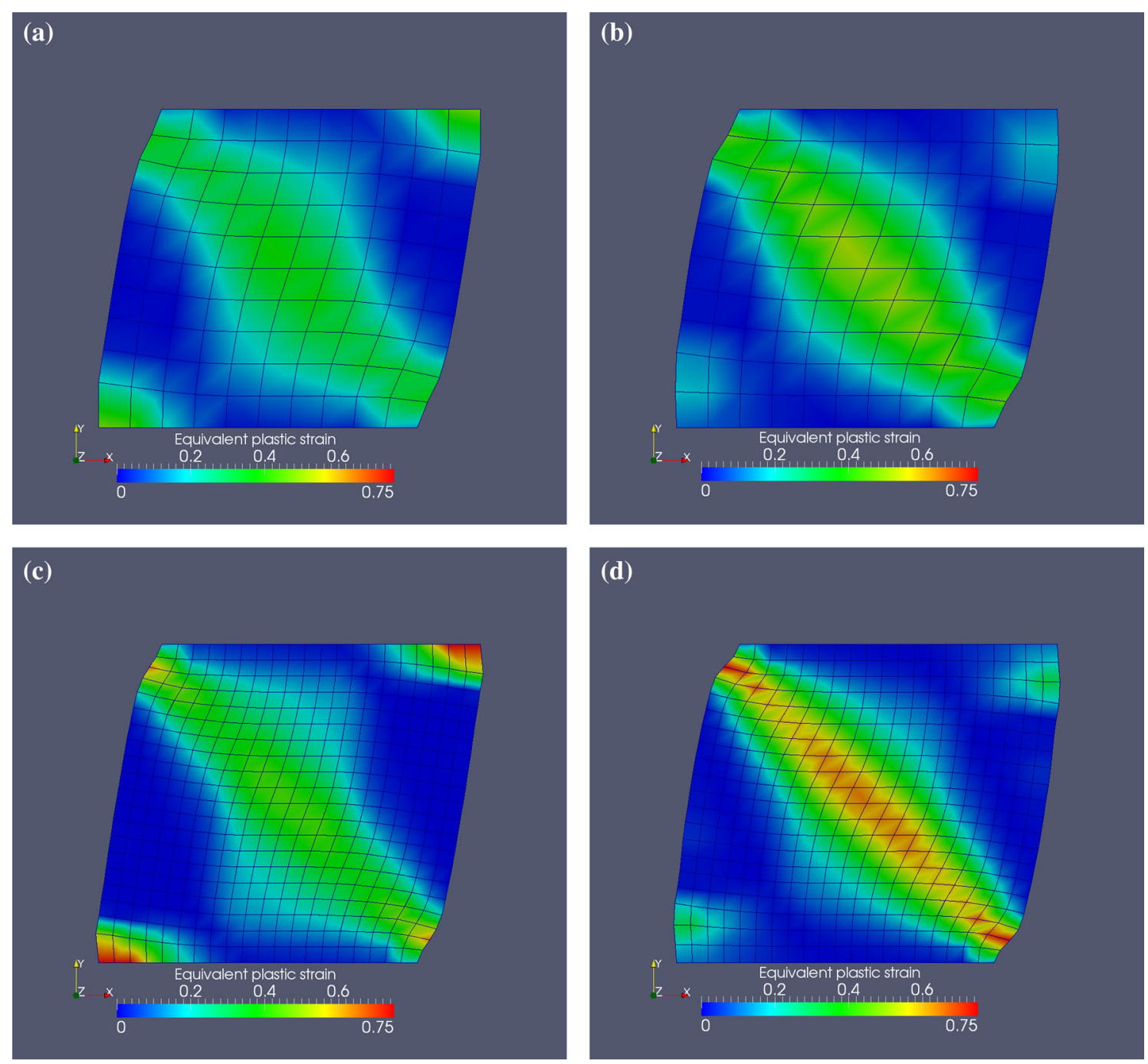

Fig. 14 Unconfined drained shear loading with non-associative cap plasticity. Equivalent plastic strain contours of fully saturated (left) and dry (right) specimens obtained from coarse and fine structured meshes at $10 \%$ global shear strain. The loading rate of the fully saturated specimen is $10^{-4} \mathrm{~m} / \mathrm{s}$. a Fully saturated specimen, b dry specimen, $\mathbf{c}$ fully saturated specimen, $\mathbf{d}$ dry specimen

400 hexahedral elements. As shown in Fig. 14b, d, we observe that the plastic response of the shear band is sensitive to mesh refinement in the dry case. In particular, the plastic zone of the fine mesh accumulates significantly more equivalent plastic strain than the coarse mesh counterpart. While a similar discrepancy can still be observed in the fully saturated specimen as shown in Fig. 14a, c, the difference on equivalent plastic strain is smaller in the shear band.

To accurately analyze how pore fluid diffusion affects the onset of strain localization, algorithms that detect a singularity in the acoustic tensor, such as those in Andrade and Borja [2], Regueiro and Foster [30], are required. This topic is out of the scope for this work, but will be considered in future studies.

\subsection{Strip punch loading}

The purpose of introducing this strip punch loading example is twofold: (1) to examine how hydro-mechanical coupling may affect the simulated mechanical and hydraulic responses and (2) to analyze how pore fluid diffusion affects the mechanical response of the shear band.

The finite element meshes and boundary conditions for the strip punch loading problem are shown in Fig. 15. The area subjected to the prescribed punch loading is $5 \mathrm{~m}$ wide and the underlying domain has a side length of $10 \mathrm{~m}$. We assume that the plane strain condition holds and the material is initially homogeneous. Body forces are neglected. Both associative and non-associative constitutive responses are simulated using the material parameters 

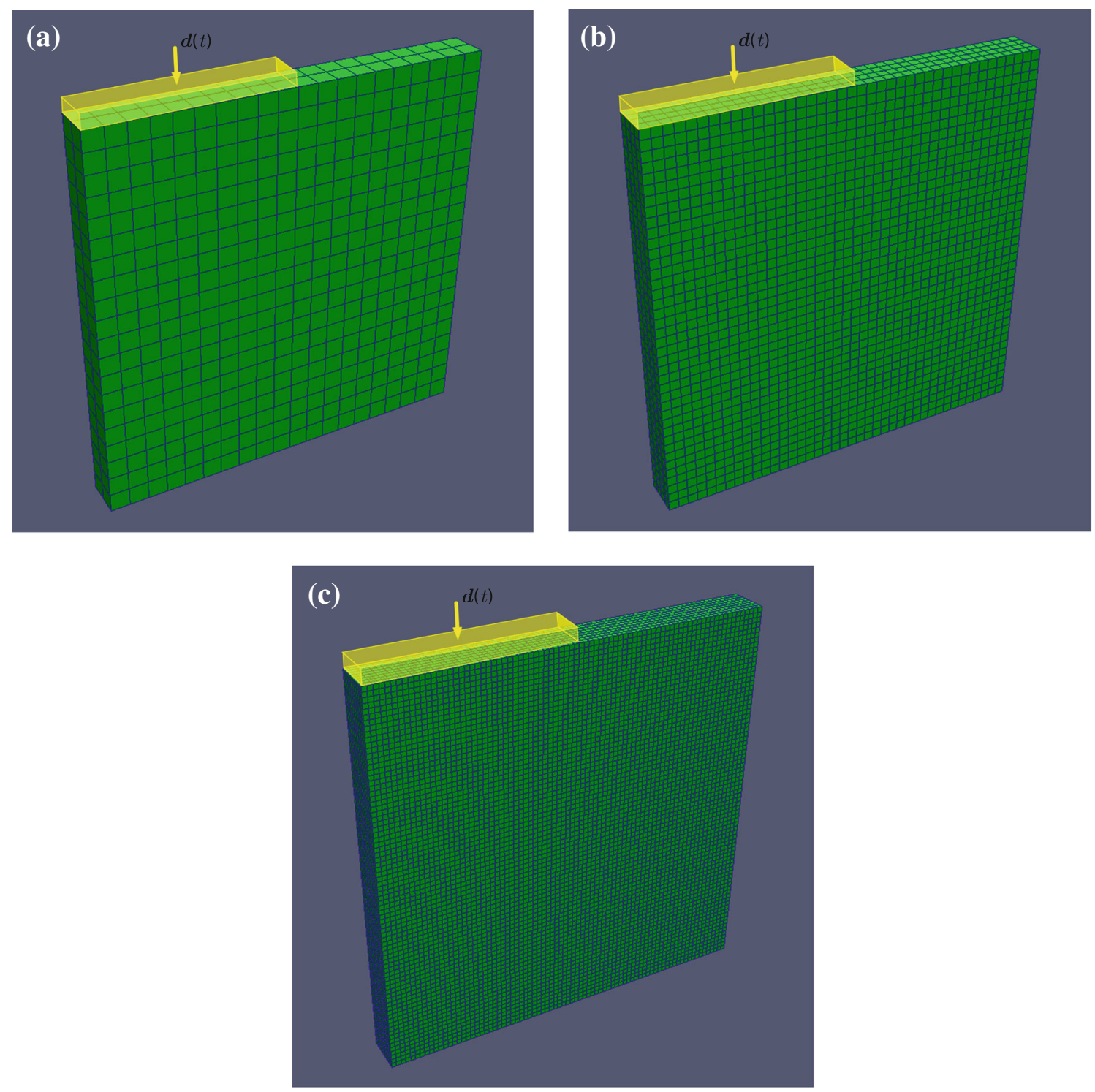

Fig. 15 Finite element meshes of the strip punch loading problem with applied displacement $\boldsymbol{d}(t)$. The top boundary is free except for the applied displacement. The right vertical side and bottom have fixed normal displacement. The left vertical side is a free surface. The top free surface has zero pressure boundary. All other surfaces have free flux boundary condition. Through thickness displacement, degrees of freedom are fixed to achieve the plain strain condition. a Number of element: 800 , b number of element: 6,400 , c number of element: 51,200

listed in Tables 1 and 2, along with the hydro-mechanical coupling parameters listed in Table 3 . We prescribe two loading rates, i.e., $10^{-6}$ and $10^{-4} \mathrm{~m} / \mathrm{s}$ to analyze the rate effect.

\subsubsection{Associative hydro-mechanical response}

In this section, we analyze the mesh sensitivity and the rate effects of the associative constitutive response in coupled hydro-mechanical simulations. Since there is no softening in the post-yielding regime, the governing equation is expected to remain well posed [30, 32]. Nevertheless, since the prescribed displacement might cause severe distortion near the tip of the strip, it is still important to quantify the gain in accuracy when consecutive mesh refinements are taken. Three finite element meshes are generated as shown in Fig. 15.

Figure 16 compares the equivalent plastic strain contours for three meshes at $t=686 \mathrm{~s}$ at a constant loading rate of $10^{-4} \mathrm{~m} / \mathrm{s}$. In all three cases, a localized band starts to form after the onset of plastic deformation. The shape and orientation of the localized bands appear insensitive to mesh refinement. Figure 17 compares the corresponding volumetric plastic strain contours. Notice that the volumetric plastic strain right at the edge of the strip is compactive (negative sign), while inside the band, it is 

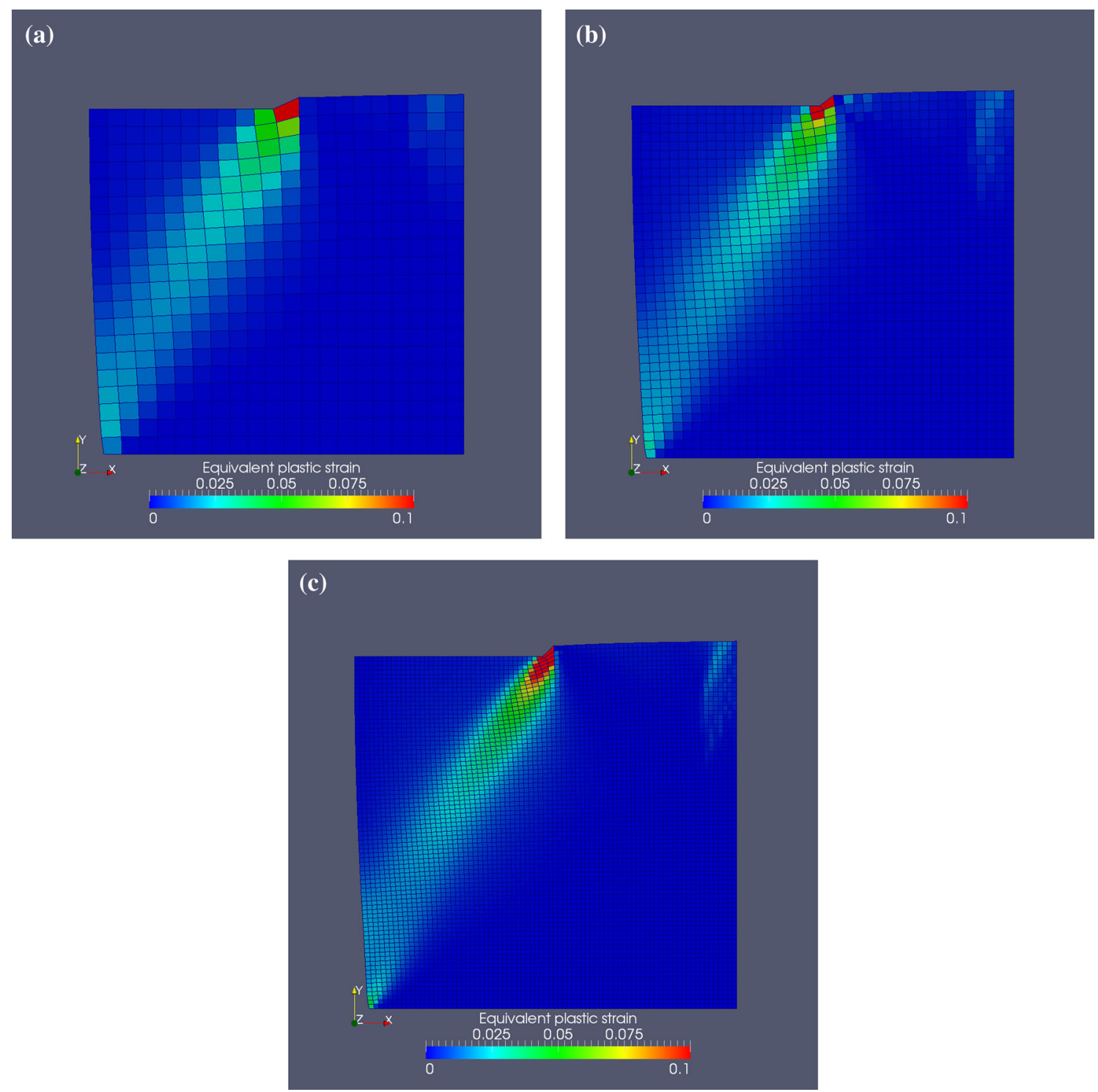

Fig. 16 Strip punch loading on a saturated limestone domain with associative cap plasticity. Equivalent plastic strain contours at time $686 \mathrm{~s}$ for three meshes at a constant loading rate of $10^{-4} \mathrm{~m} / \mathrm{s}$. The displacement field is scaled by a factor of 5

dilative (positive sign). Figure 18 compares the corresponding pore pressure contours. All three meshes give similar pore pressure distributions.

The global load-displacement response of the strip punch loaded at a rate of $10^{-4} \mathrm{~m} / \mathrm{s}$ for each mesh resolution is shown in Fig. 19. It can be seen that the responses are very similar, and as the mesh is refined, the resultant force decreases slightly. Figure 20 shows the nodal pore pressure time histories at the middle of the left vertical surface obtained from the coarse and refined meshes. The pore pressure time histories, as shown in Fig. 20, are practically indistinguishable. According to the results shown in Figs. 17 and 18, the size and location of the plastic zone and the pore pressure distribution are both insensitive to mesh refinement.
To analyze the local effective stress response at various locations, we select three representative points, shown in Fig. 21, and record the corresponding stress paths during the simulations. Note that the predicted responses at the tip of the punch loading are highly sensitive to the mesh size as demonstrated previously in Van-Langen and Vermeer [49]. While introducing a zero-thickness interface element or other enhancements that enable strong discontinuity in displacement may significantly improve the accuracy of the responses at the tips, these enhancements are outside the scope of this work. As a result, we do not analyze the stress-strain response of the element adjacent to the punch loading.

Figure 22 shows the effective stress path at the three selected points at loading rate $10^{-4} \mathrm{~m} / \mathrm{s}$ in two meridional 

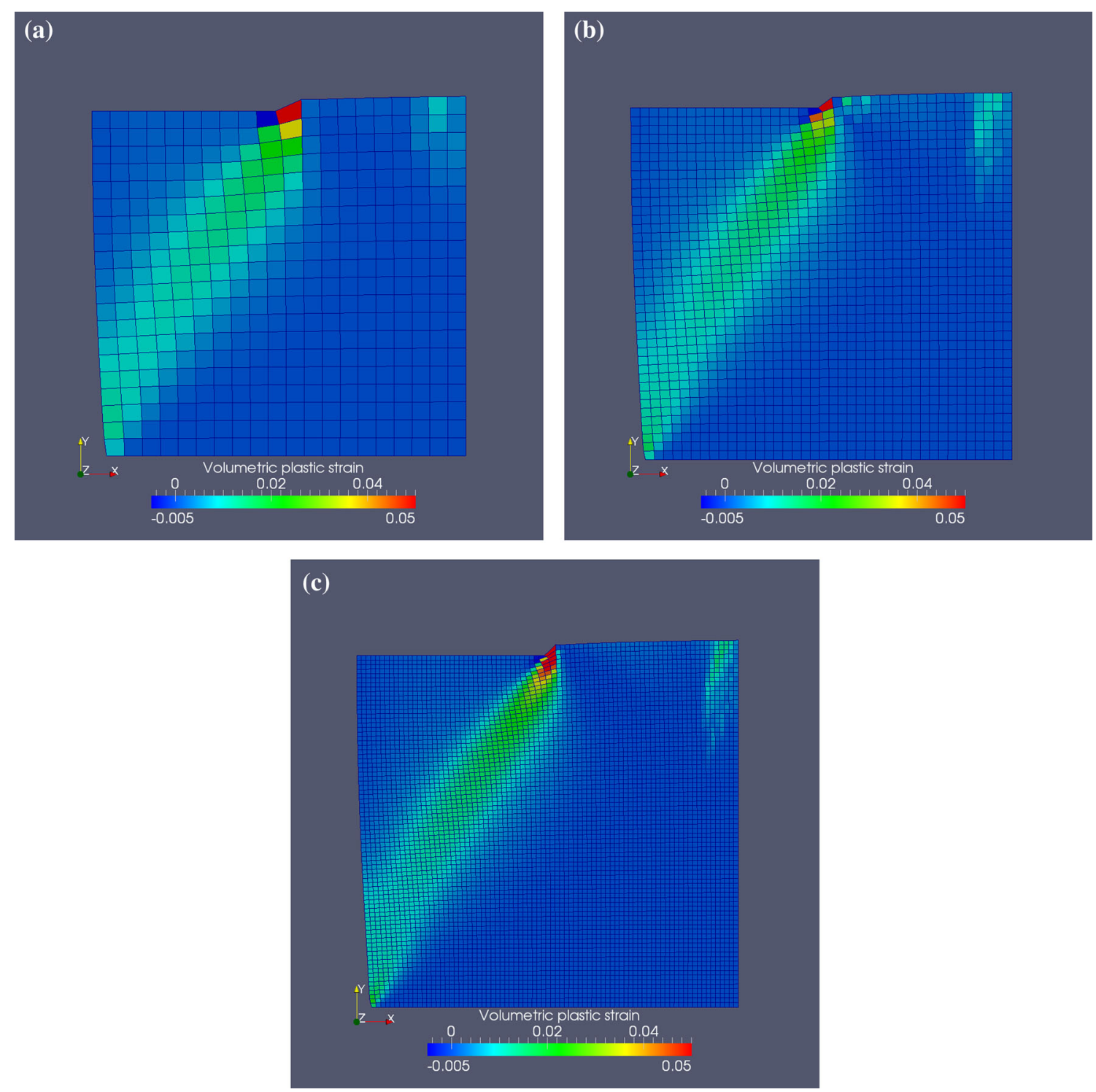

Fig. 17 Strip punch loading on a saturated limestone with associative cap plasticity. Volumetric plastic strain contour at $t=686 \mathrm{~s}$ for three meshes at a constant loading rate of $10^{-4} \mathrm{~m} / \mathrm{s}$. The displacement field is scaled by a factor of 5

stress spaces, i.e. a $\sqrt{J_{2}^{\xi}}$ versus $I_{1}$, and $\mathbf{b} \sqrt{J_{2}}$ versus $I_{1}$. According to Fig. 22a, all three locations initially yield at the shear part of the yield surface. As the loading continues, Points 2 and 3 stay on the shear yield surface, while Point 1 starts to move toward the compactive (cap) portion of the yield surface. This indicates that the plastic deformation of the material at Point 1 transitions from a sheardilatant dominated mechanism to a shear-compactive dominated mechanism.

Figure $22 \mathrm{~b}$ shows the failure surface of Point 1 at the end of the simulation. Notice that the final stress state of Point 1 is approaching the compactive part of the failure surface. The corresponding shear stress-strain behavior of the selected points is plotted in Fig. 23. Notice that Point 1 undergoes significantly larger shear stain compared to the other two points. This observation is consistent with the equivalent strain contours in Fig. 21 showing the concentration of shear strain near the edge of the punch loading. None of the points has reached the failure surface yet, and thus, the perfect plasticity is not observed in the shear stress-strain response.

Figure 24a compares the effective stress near the edge of the strip for two loading rates. According to Fig. 24a, plastic yielding occurred at lower mean effective stress when loading is prescribed at a faster rate. This difference in initial yielding can be explained by the fact that 

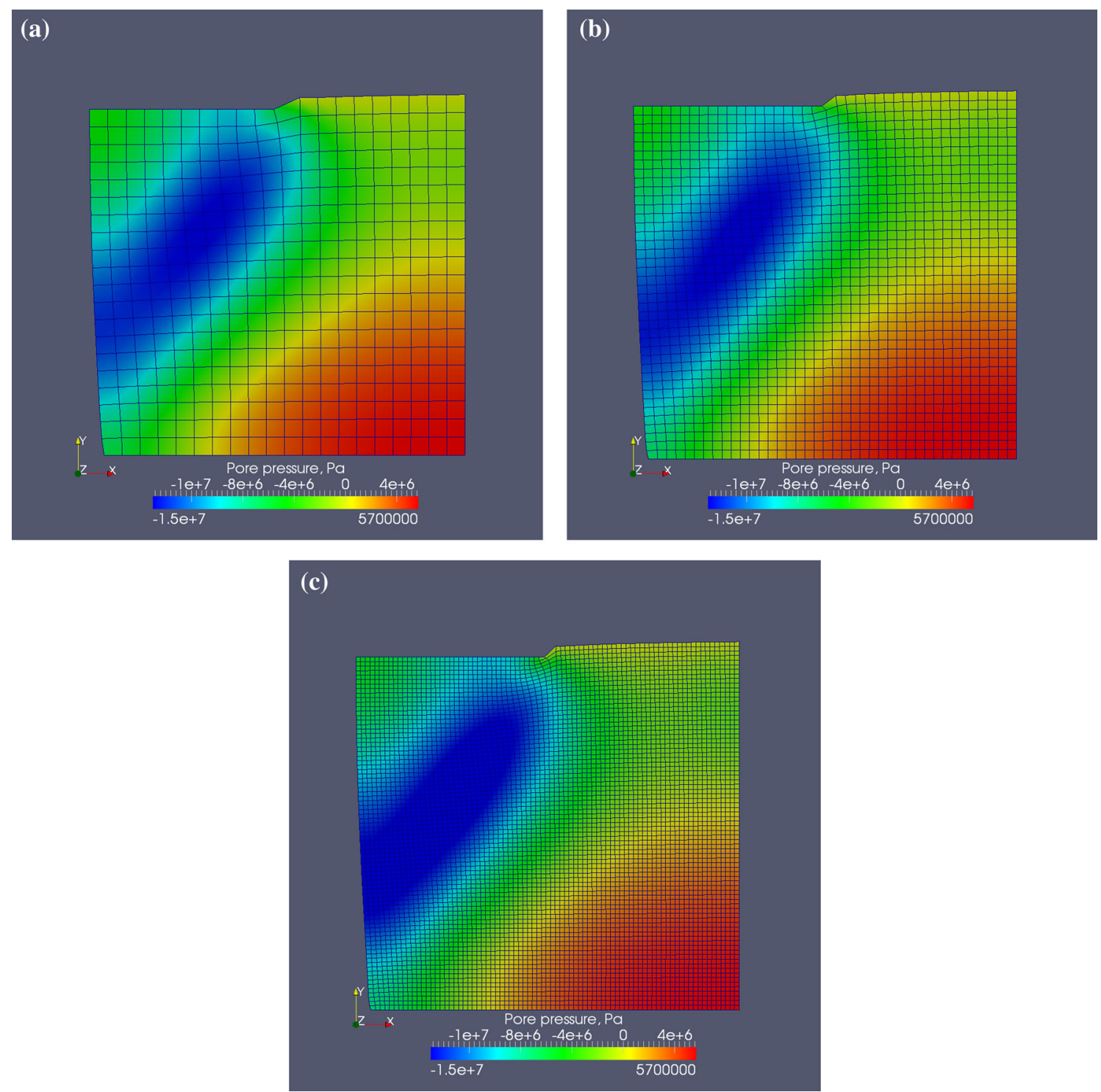

Fig. 18 Strip punch loading on a saturated limestone with associative cap plasticity. Pore pressure contour at $t=686 \mathrm{~s}$ for three meshes at a constant loading rate of $10^{-4} \mathrm{~m} / \mathrm{s}$. The displacement field is scaled by a factor of 5

the higher loading rate limits the dissipation of pore pressure and causes more excess pore pressure induced by skeleton deformation. This higher pore pressure, which is shown in Fig. 25a, in turn leads to lower mean effective stress and causes the geomaterial to yield on the shear-dilatational side of the surface at a lower mean effective stress.

Figure $24 \mathrm{~b}$ compares the porosity evolution of the material at Point 1 . While the material at Point 1 exhibits porosity reduction in both cases, the porosity reduction is more significant in the fast loading rate case. As shown in Fig. 25, this is attributed to both the compactive plastic volumetric strain and the substantial amount of negative pore pressure that lead to changes in densities of the compressible solid and fluid constituents.

\subsubsection{Non-associative hydro-mechanical response}

As reported in [30], the associative version of the cap plasticity model does not lead to the loss of ellipticity. As a result, both the single-phase dry and hydro-mechanical responses are not prone to exhibit mesh dependence.

In an attempt to analyze whether the hydro-mechanical coupling regularizes the system, we repeat the previous boundary value problems with non-associative cap plasticity parameters, as listed in Table 2 . Our objective is to compare the non-associative response of the single-phase dry simulations with the water-saturated counterparts and analyze the effects of hydro-mechanical coupling. Boundary conditions and geometry are identical with those used in the previous associative plasticity example. 


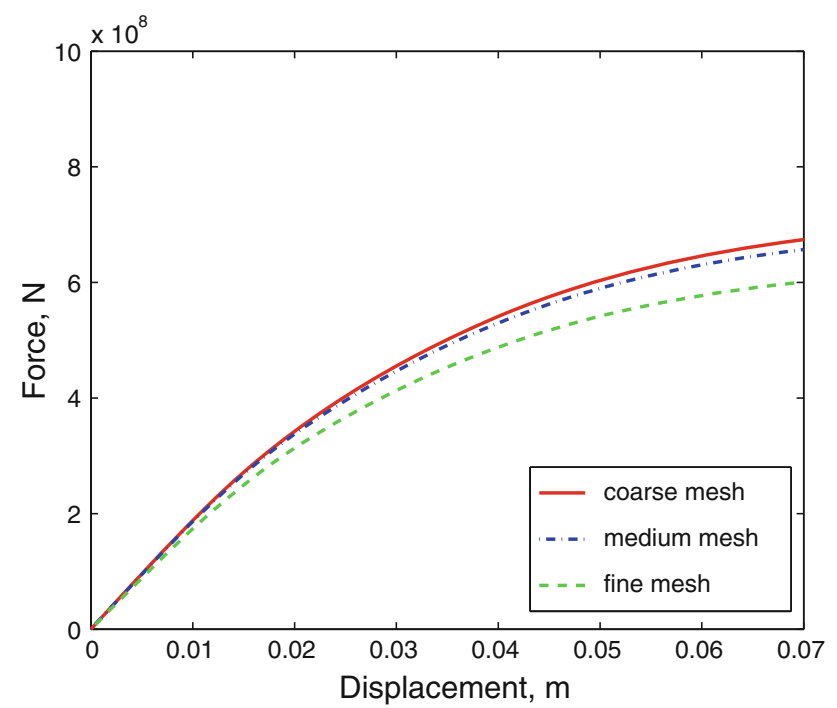

Fig. 19 Strip punch loading on a saturated limestone domain with associative cap plasticity. Global load-displacement responses with a prescribed $10^{-4} \mathrm{~m} / \mathrm{s}$ loading rate

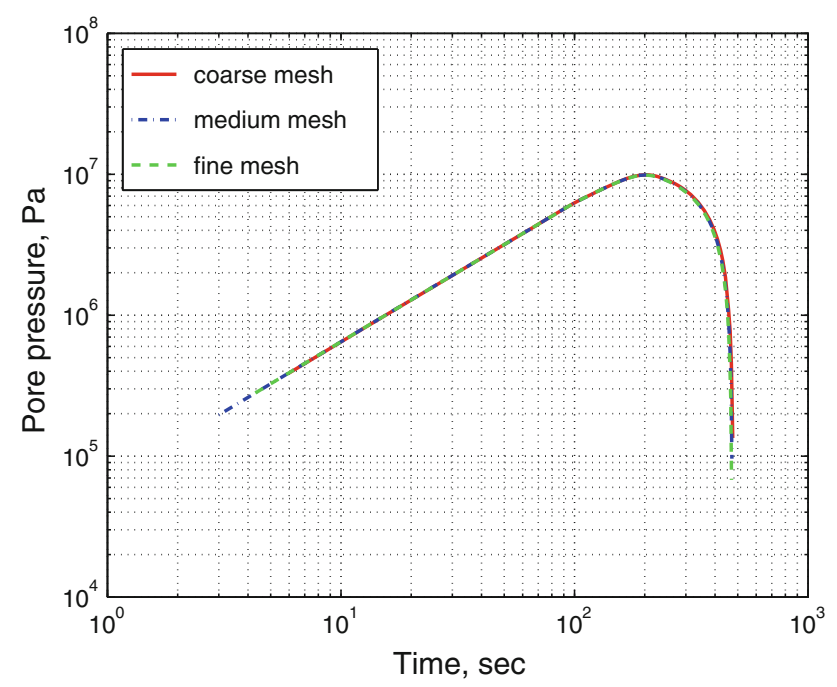

Fig. 20 Strip punch loading on a saturated limestone domain with associative cap plasticity. Time histories of pore pressure (log scale) for the node at the middle of the left vertical surface for loading rate $10^{-4} \mathrm{~m} / \mathrm{s}$

The prescribed loading rate is $10^{-4} \mathrm{~m} / \mathrm{s}$. We first compare the shear stress-strain responses of the three selected points shown in Fig. 21. Interestingly, from Fig. 26, we found that existence of pore fluid does not impose any significant difference on the shear response in Points 2 and 3. By contrast, the shear stress-strain response at Point 1 (the tip) is more sensitive to the drainage condition. In particular, we observe a slight shear stress drop after reaching a local peak at $80 \mathrm{MPa}$. Notice that the shear stress is monotonically increasing during the dry simulation. Furthermore, the shear stress at the end of the fully

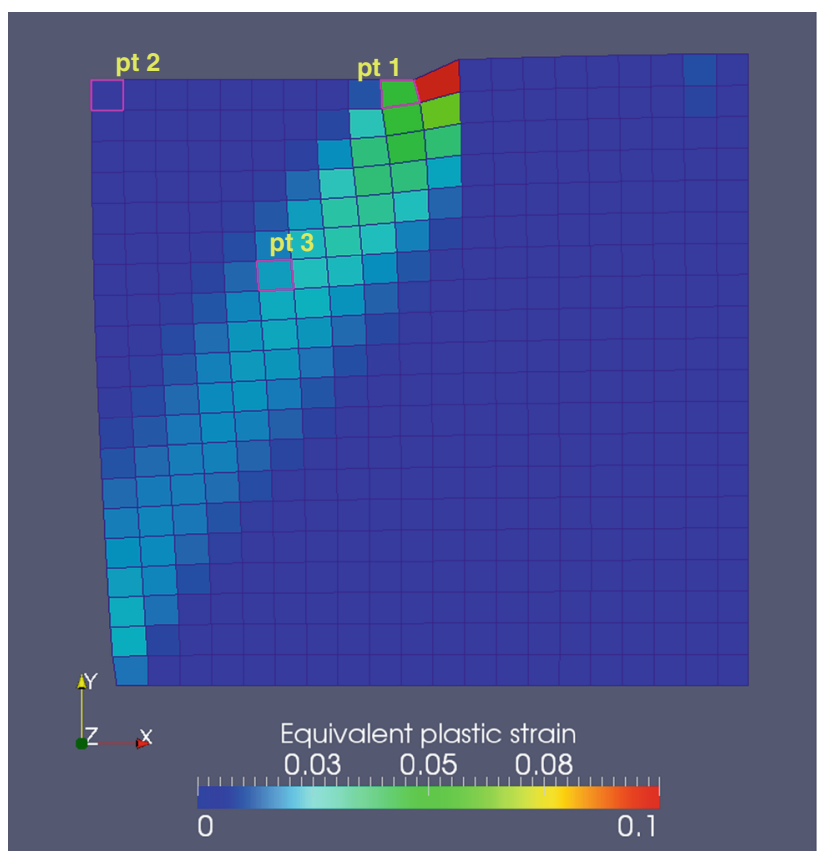

Fig. 21 Strip punch loading on a saturated limestone domain with associative cap plasticity. Equivalent plastic strain contours showing locations of three selected points (Gauss points within highlighted elements) for reporting stress path. Contour plotted at $t=686 \mathrm{~s}$ at a constant loading rate of $10^{-4} \mathrm{~m} / \mathrm{s}$

saturated simulation is approximately $10 \%$ less than the dry counterpart. Figure 27 shows the stress path at the tip of the punch loading. The pore fluid diffusion, which is prescribed by a zero pore pressure, is significant enough to cause a noticeable shift of the initial yield point in both the $\sqrt{J_{2}^{\xi}}$ versus $I_{1}$ and $\sqrt{J_{2}}$ versus $I_{1}$ stress space. In turn, this shift leads to less isotropic hardening in the fully saturated material and therefore lowers the shear stress in the post-yielding regime. In contrast, Fig. 28 shows the stress path of Point 3, which is located inside the localization band. Unlike the stress path of Point 1, the stress of Point 3 stays on the shear part of the yield surface. The yield surface of Point 3 does not enlarge significantly. This lack of significant hardening is also observed in the octahedral shear stress versus octahedral shear strain curves in Fig. 26. Since the prescribed loading is progressing at a constant rate, excess pore pressure is not expected to be fully dissipated at Point 2 and Point 3 . The responses shown in Figs. 26 and 28 nevertheless indicate that the excess pore pressure seems to have little effect in regions far away from the edge of the punch load. On the other hand, the excess pore pressure at Point 1 is expected to be small due to its close proximity to the zero pore pressure surface. However, we still observe a measurable difference in octahedral shear stress of dry and fully saturated materials, as shown in Figs. 26 and 27. 


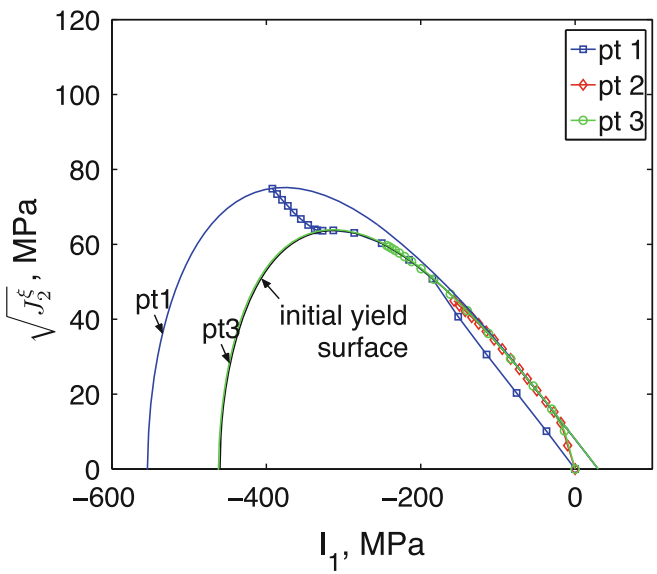

(a)

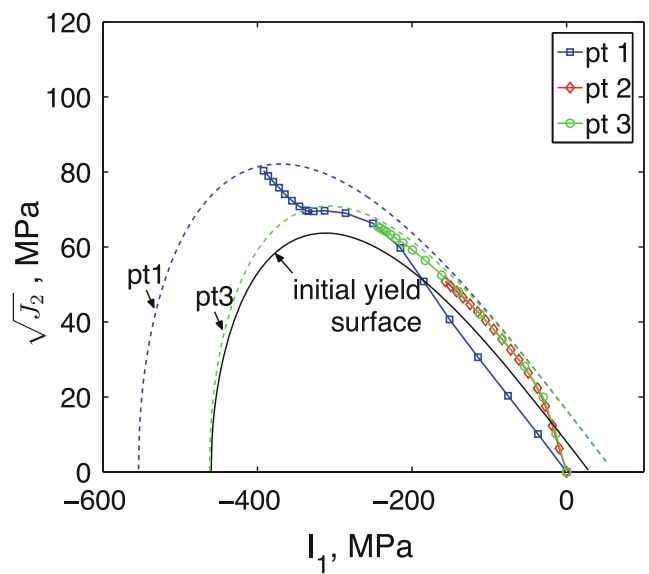

(b)

Fig. 22 Strip punch loading on a saturated limestone domain with the associative cap plasticity model. a Effective stress path in meridional stress space $\sqrt{J_{2}^{\xi}}$ versus $I_{1}$, solid lines (marked pt 1 and pt 3 ) showing final yield surfaces; b effective stress path in meridional stress space $\sqrt{J_{2}}$ versus $I_{1}$, dashed lines (marked pt 1 and pt 3) showing final failure surfaces; The prescribed loading rate is $10^{-4} \mathrm{~m} / \mathrm{s}$

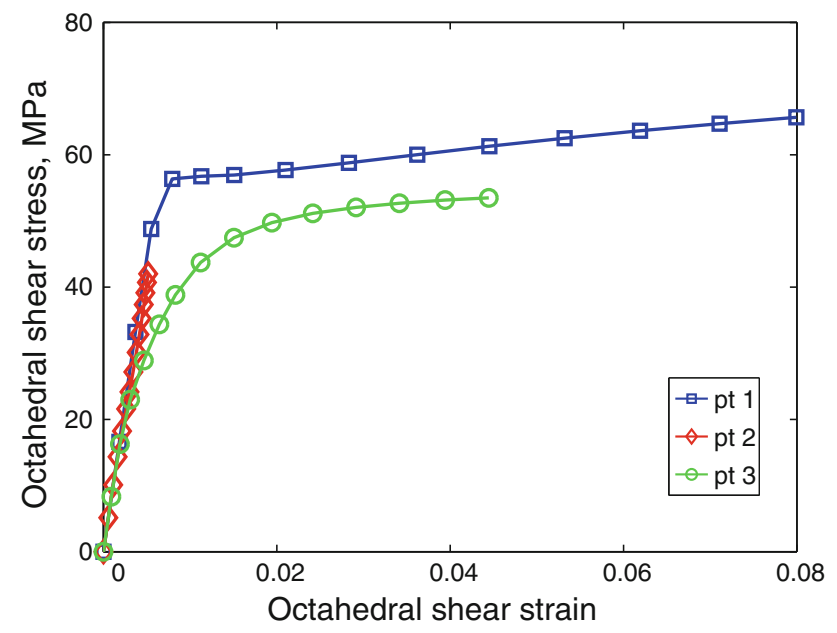

Fig. 23 Strip punch loading on a saturated limestone domain with associative cap plasticity. Octahedral shear stress-strain responses at the three selected points from simulations with a $10^{-4} \mathrm{~m} / \mathrm{s}$ loading rate

To assess the mesh sensitivity of the hydro-mechanical responses, we conduct refinement studies on both dry and fully saturated cases. Figures 29 and 30 show the equivalent plastic strain and volumetric plastic strain of the dry and fully saturated materials with three levels of consecutive mesh refinement. As pore fluid diffusion may dissipate part of the energy, we find that the plastic zone developed in the fully saturated simulations tends to be larger and more diffusive.

Figure 31 compares the global force-displacement responses for three meshes, in (a) dry and (b) fully saturated conditions. Interestingly, we found that the global force-displacement responses seem to be insensitive to the refinement of the finite element meshes when only a small amount of plastic strain accumulates in the plastic zone. This insensitivity to mesh refinement is observed even when plastic strain is concentrated in a localized region.

Presumably, the discrepancies among various meshes could possibly be more apparent if the materials are further loaded in the post-bifurcation regime. However, since the simulations fail to converge when the prescribed displacement is above $0.07 \mathrm{~m}$, our simulations stopped prematurely before more plastic strain accumulates. Since loss of ellipticity is not checked, it is unclear whether bifurcation(s) occurs. Algorithms that detect bifurcations, such as those in Andrade and Borja [2], Regueiro and Foster [30], are outside the scope of this work, but will be considered in future studies.

\subsection{Three-dimensional rigid circular punch loading}

The purpose of this three-dimensional simulation is to examine the influence of the hydro-mechanical coupling effect on the mechanical and hydraulic responses when the porous medium is close to the drained limit. A threedimensional circular punch load with $3 \mathrm{~m}$ radius is applied on saturated collapsible limestone block. The limestone block is $30 \mathrm{~m}$ in radius and $30 \mathrm{~m}$ in height. Since the circular domain allows the pore fluid to flow in more directions than the strip domain, the rate of consolidation is expected to be higher than those in the previous examples. The mechanical and hydraulic responses of the limestone are modeled via the associative version of the cap plasticity model with material parameters listed in Tables 1 and 3.

The bottom of the limestone domain is fixed and a linear ramp function $\mathrm{d}(t)$ is used to prescribe the vertical displacement of the punch area at different loading rates. Furthermore, displacements in the normal direction of the 


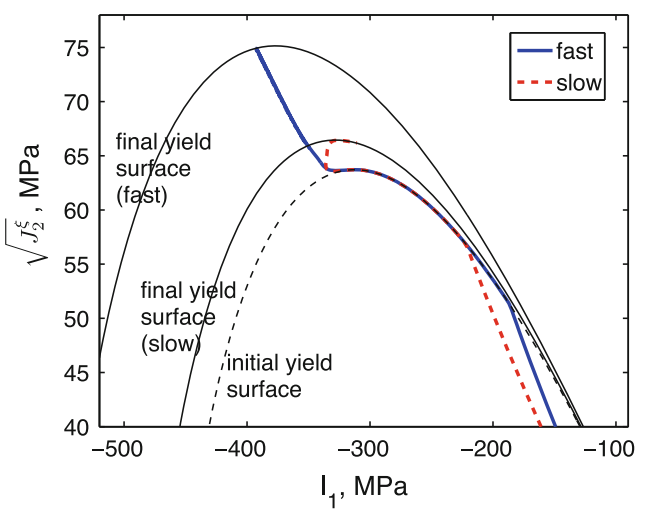

(a)

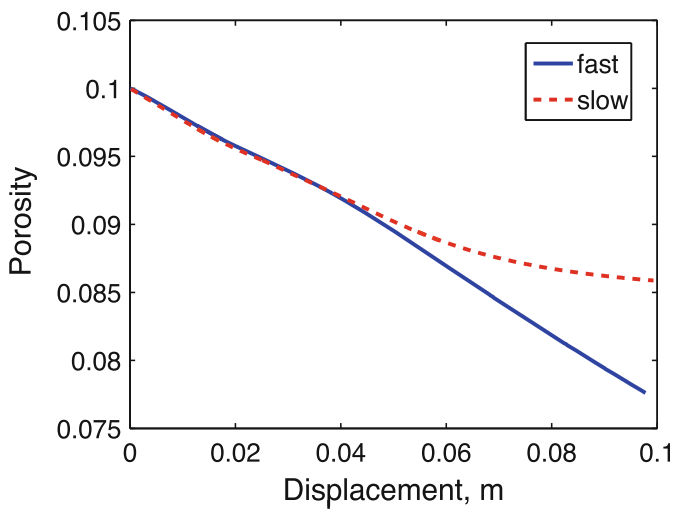

(b)

Fig. 24 Strip punch loading on a saturated limestone domain with associative cap plasticity. a Effective stress path in meridional stress space $\sqrt{J_{2}^{\xi}}$ versus $I_{1}$ and $\mathbf{b}$ porosity evolution of a material point near the edge of the strip loading (pt 1 shown in Fig. 21) for two loading rates, denoted as fast $\left(10^{-4} \mathrm{~m} / \mathrm{s}\right)$ and slow $\left(10^{-6} \mathrm{~m} / \mathrm{s}\right)$

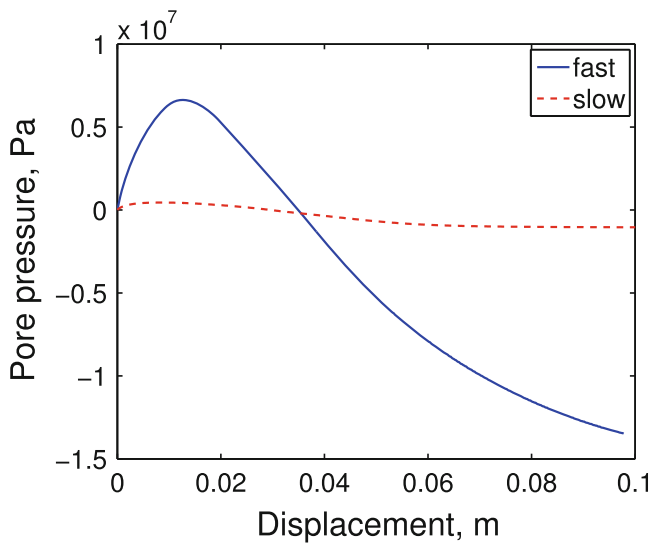

(a)

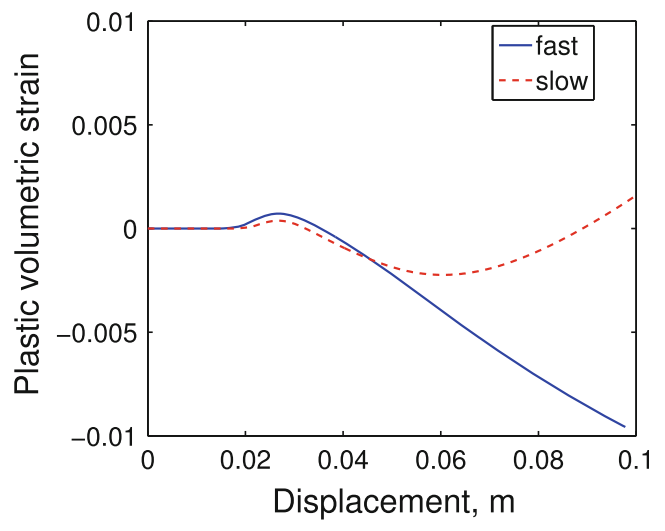

(b)

Fig. 25 Strip punch loading on a saturated limestone domain with associative cap plasticity. a Pore pressure and b plastic volumetric strain of a material point near the edge of the strip loading (pt 1 shown in Fig. 21) for two loading rates, denoted as fast $\left(10^{-4} \mathrm{~m} / \mathrm{s}\right)$ and slow $\left(10^{-6} \mathrm{~m} / \mathrm{s}\right)$

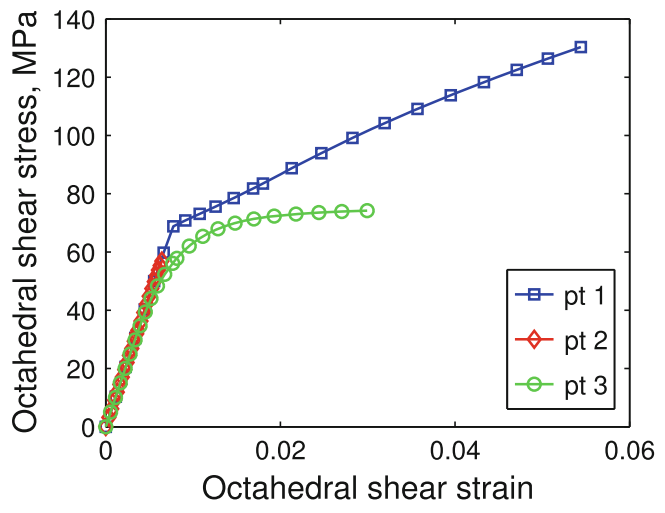

(a)

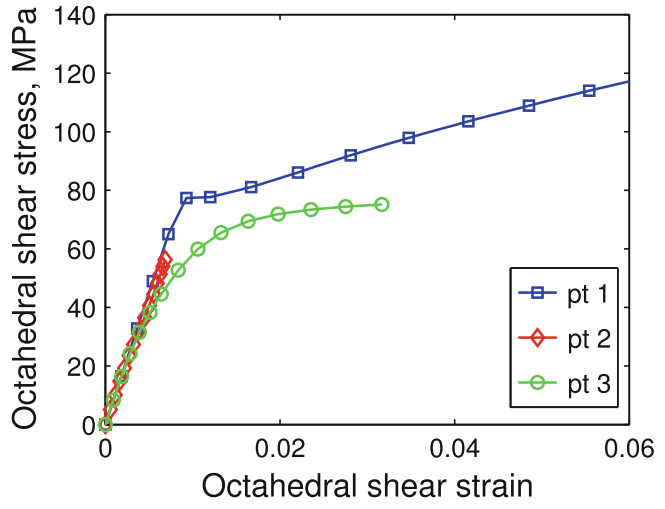

(b)

Fig. 26 Strip punch loading with non-associative cap plasticity. Octahedral shear stress-strain response at the three selected points (shown in Fig. 21) from dry and fully saturated simulations with a $10^{-4} \mathrm{~m} / \mathrm{s}$ loading rate. a Non-associative single-phase response, b non-associative hydromechanical response 


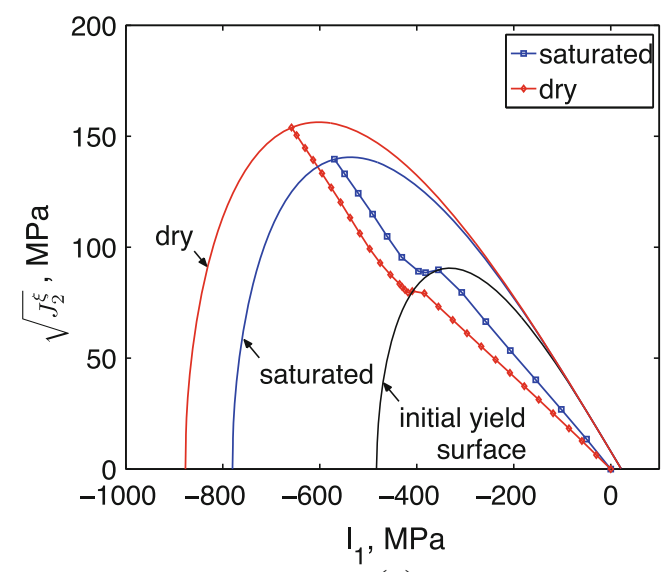

(a)

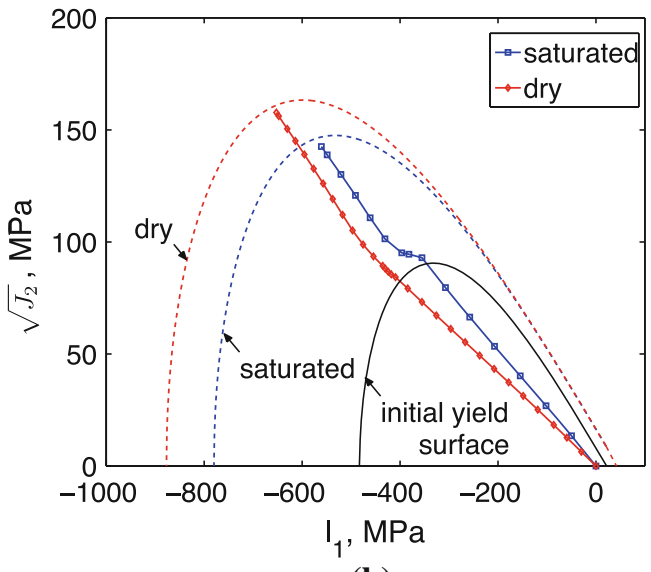

(b)

Fig. 27 Strip punch loading with a non-associative cap plasticity model. a Effective stress path for pt 1 in meridional stress space $\sqrt{J_{2}^{\xi}}$ versus $I_{1}$, solid lines (marked dry and saturated) showing final yield surfaces; b effective stress path for pt 1 in meridional stress space $\sqrt{J_{2}}$ versus $I_{1}$, dashed lines (marked dry and saturated) showing final failure surfaces. The prescribed loading rate is $10^{-4} \mathrm{~m} / \mathrm{s}$. Location of pt 1 is shown in Fig. 21

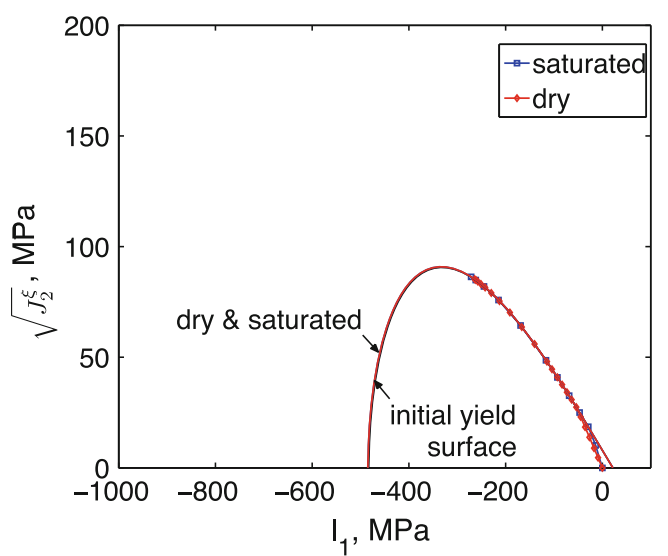

(a)

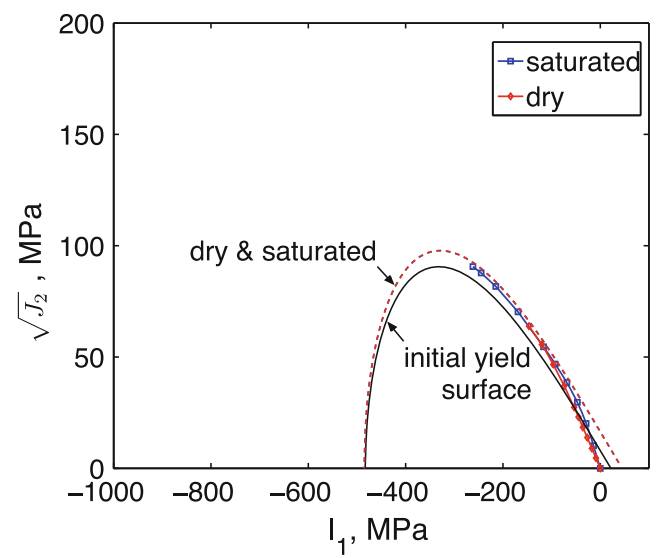

(b)

Fig. 28 Strip punch loading with a non-associative cap plasticity model. a Effective stress path for pt 3 in meridional stress space $\sqrt{J_{2}^{\xi}}$ versus $I_{1}$, solid lines (marked dry and saturated) showing final yield surfaces; b effective stress path for $\mathrm{p} 3$ in meridional stress space $\sqrt{J_{2}}$ versus $I_{1}$, dashed lines (marked dry and saturated) showing final failure surfaces. The prescribed loading rate is $10^{-4} \mathrm{~m} / \mathrm{s}$. Location of pt 3 is shown in Fig. 21

two orthogonal surfaces of the limestone domain, at radius $r=30 \mathrm{~m}$, are prescribed to be zero, while the top free surface is traction free.

For the pore pressure, the bottom of the limestone domain is given a zero-flux boundary condition. The two orthogonal surfaces of the limestone domain, at radius $r=30 \mathrm{~m}$, as well as the side of the cylindrical domain, are also considered zero-flux boundaries. The pore pressure of the top surface is prescribed as zero, except underneath the punch area, to simulate the fully drained surface.

A non-uniform, equal-order finite element mesh, as shown in Fig. 32, is used. Notice that a very fine discretization is used at the edge of the circular punch and is necessary to adequately resolve the sharp stress gradient at the edge. Figure 33 shows the effective vertical stress along the two orthogonal edges of the circular punch, where sharp gradients are observed. Different loading rates, $0.2,0.02$, and $0.002 \mathrm{~m} /$ day are prescribed in this example. Figure 34 shows the pore pressure distribution for the three different loading rates at the same applied displacement. As expected, at higher loading rates higher pore pressure accumulates near the tip of the punch. Unlike the 2D case, the pore pressure build-up near the punch tip is several orders lower than the effective stress for loading rates 0.02 and $0.002 \mathrm{~m} /$ day and therefore closer to the drained limit. This result is attributed to the fact that the multiple flow 

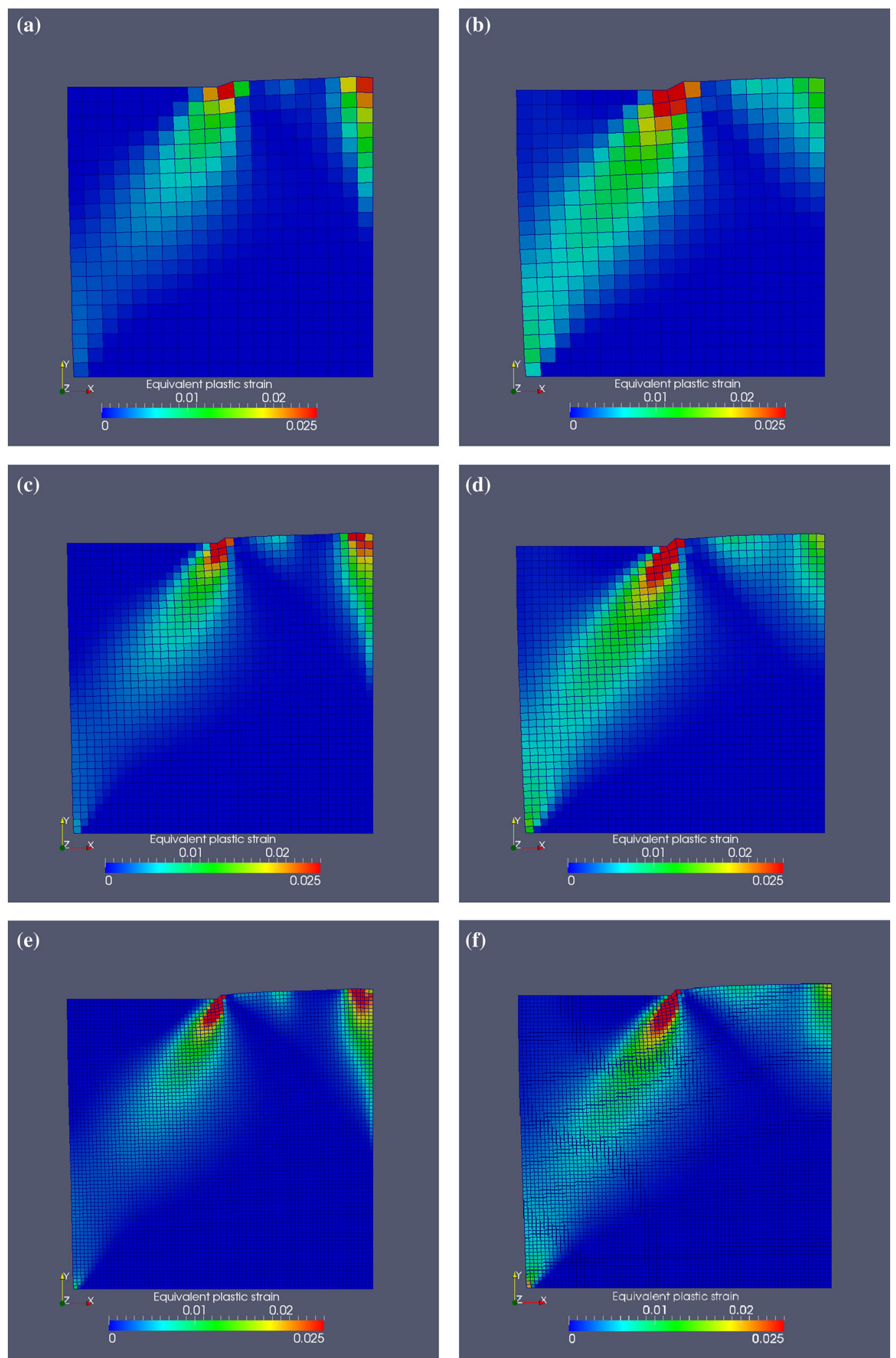

Fig. 29 Strip punch loading on dry (left column) and saturated (right column) domains with non-associative cap plasticity. Equivalent plastic strain contours at $t=642 \mathrm{~s}$. The displacement field is scaled by a factor of 5. a Dry, mesh 1, b saturated, mesh 1, c dry, mesh 2, d saturated, mesh 2 , e dry, mesh 3 , f saturated, mesh 3 

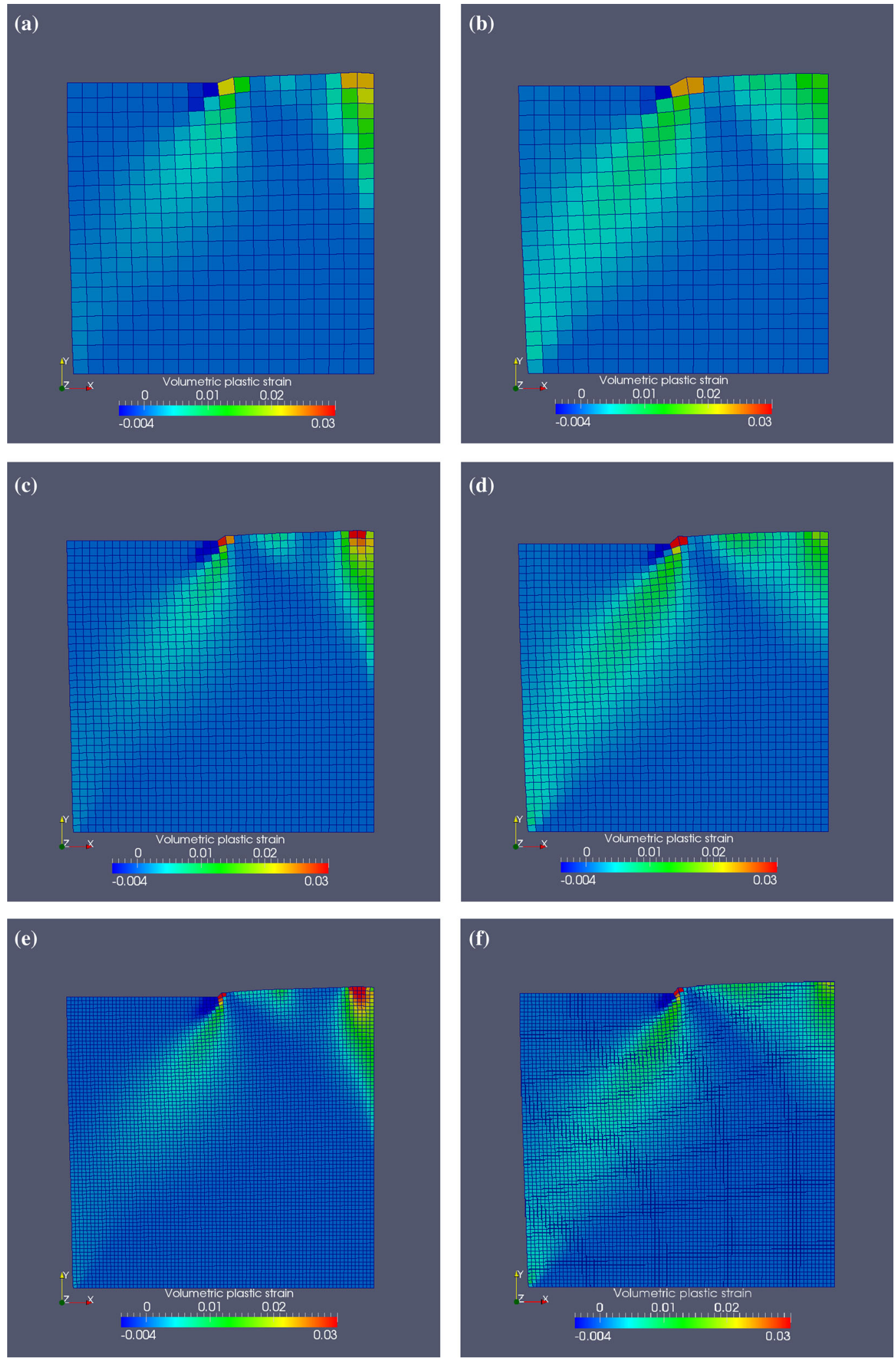

Fig. 30 Strip punch loading on dry (left column) and saturated (right column) domains with non-associative cap plasticity. Volumetric plastic strain contours at $t=642 \mathrm{~s}$. The displacement field is scaled by a factor of 5 . a Dry, mesh 1, b saturated, mesh 1, c dry, mesh 2, d saturated, mesh 2 , e dry, mesh 3, f saturated, mesh 3 


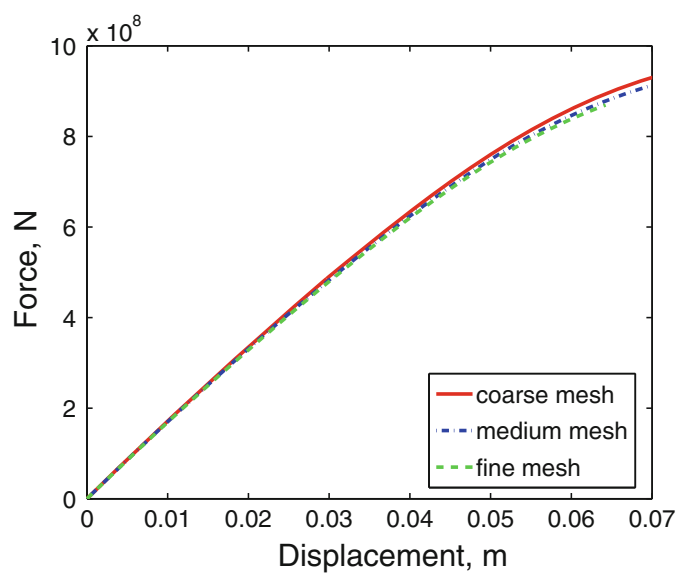

(a)

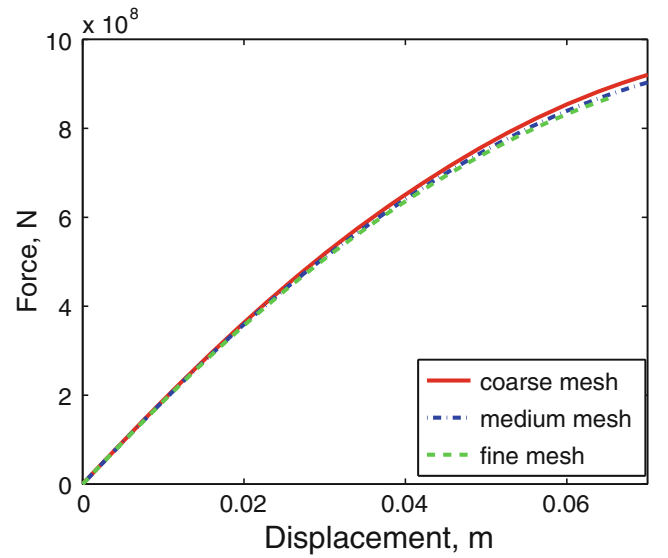

(b)

Fig. 31 Strip punch loading with non-associative cap plasticity. Global load-displacement responses for: a saturated geomaterials; b dry geomaterials. a Non-associative single-phase response, b non-associative hydro-mechanical response

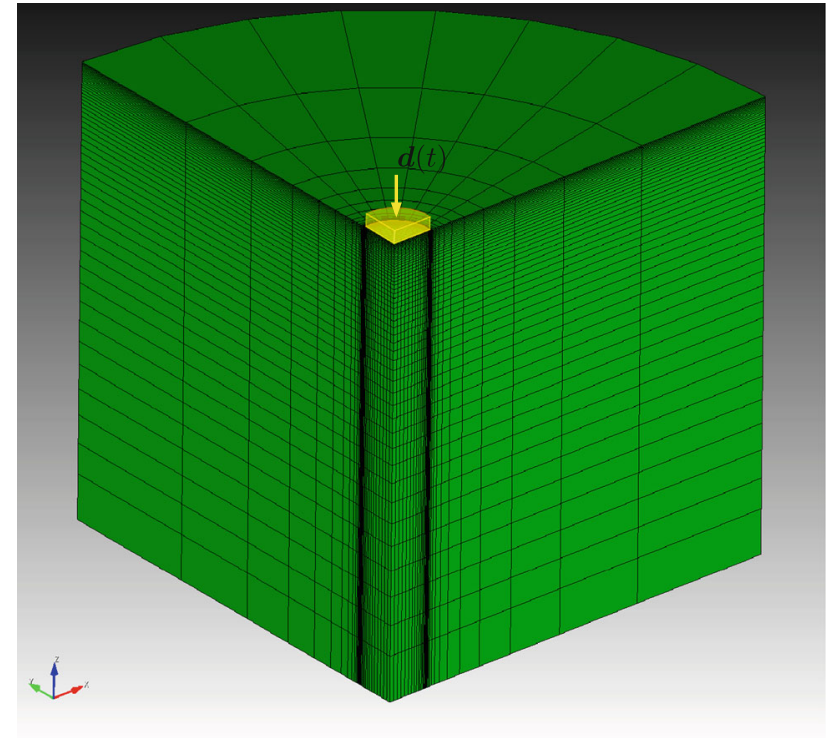

Fig. 32 Finite element mesh of three-dimensional rigid circular punch with applied displacement $\boldsymbol{d}(t)$. The top boundary is free except for applied displacement at the center. The sides have fixed normal displacement due to symmetry, while the bottom has fixed normal displacements

directions available in the circular domain help dissipating pore pressure.

Figure 35 compares the pore pressure along the edge of the domain obtained at three prescribed loading rates. Again, we observe that faster loading rates result in higher pore pressure and also a sharper pore pressure gradient. This sharper pore pressure gradient in turn leads to higher pore fluid relative velocity. The substantial amount of diffusion therefore prevents the low compressibility of the

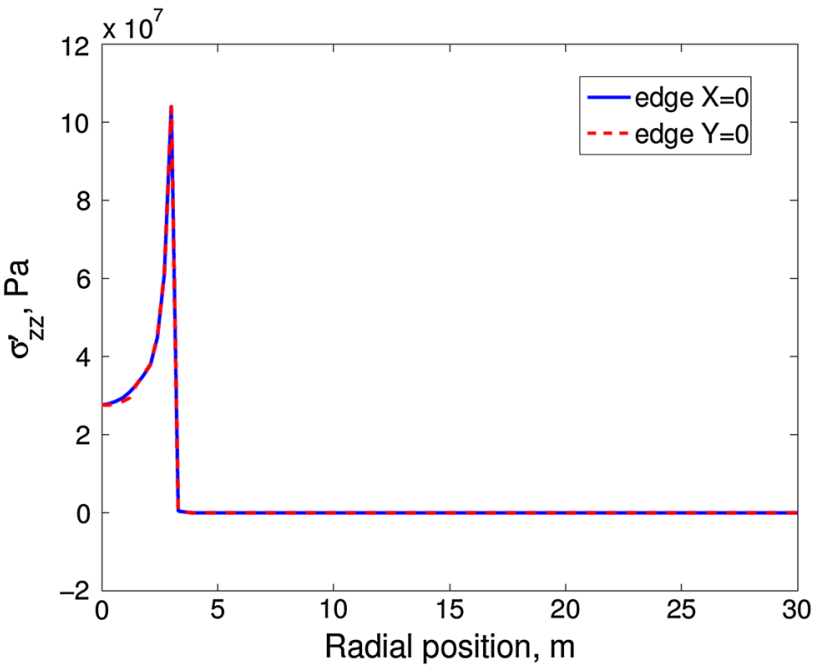

Fig. 33 Three-dimensional punch loading with associative cap plasticity. Effective stress profile (vertical component) along the two orthogonal edges of the circular punch for the 1-day loading rate. Sharp stress gradients are captured using the refined discretization close to the edge. Radial position $r=0 \mathrm{~m}$ is at the center of the circular punch

pore fluid constituent from constraining the volumetric deformation of the solid skeleton. Figure 36 plots the pore pressure and porosity evolution for a selected Gauss point at the edge of the punch (Point 1 in Fig. 38). In the fastest case $(0.2 \mathrm{~m} /$ day $)$, the high pore pressure constrains the volumetric deformation and hence results less porosity reduction. In the slowest case $(0.002 \mathrm{~m} /$ day $)$, while the presence of pore fluid may still reduce the volumetric deformation of the solid skeleton, the porosity reduction is more severe. This notable difference in porosity reduction 

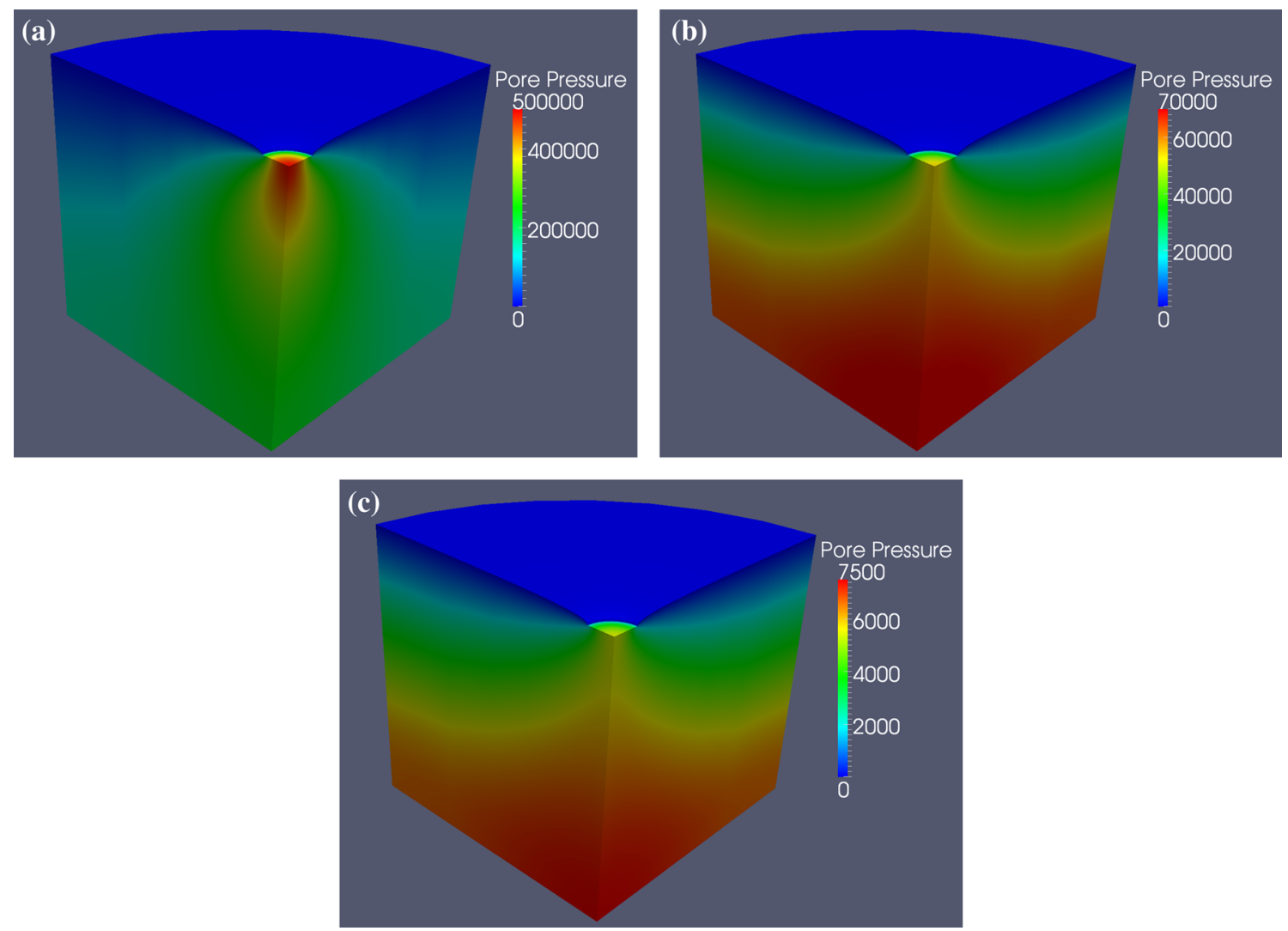

Fig. 34 Three-dimensional punch loading with associative cap plasticity. Pore pressure contours for three different loading rates at prescribed displacement $d=9.52 \times 10^{-3}$ ] $\mathrm{m}$. Units in the figures are Pa. a $0.2 \mathrm{~m} /$ day loading rate, $\mathbf{b} 0.02 \mathrm{~m} /$ day loading rate, $\mathbf{c} 0.002 \mathrm{~m} /$ day loading rate

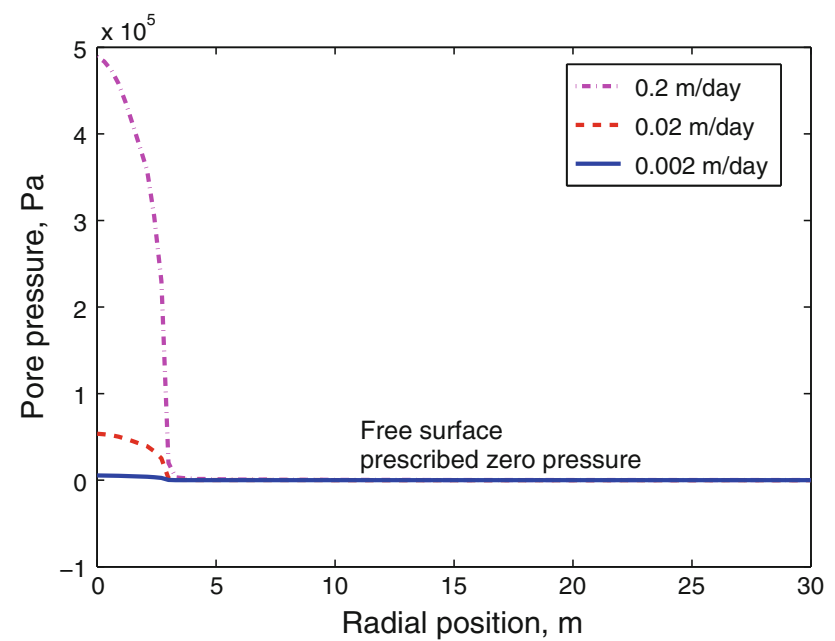

Fig. 35 Three-dimensional punch loading with associative cap plasticity. Pore pressure along the edge developed at three different loading rates. Prescribed displacement $d=9.52 \times 10^{-3} \mathrm{~m}$. Units in the figures are $\mathrm{Pa}$. Radial position $0 \mathrm{~m}$ is at the center of the punch

is an interesting result, given the fact that the pore pressure is much smaller than the effective stress in all three cases. Conventionally, this small excess pore pressure is thought to have little influence on the mechanical response. However, due to the plastic yielding on the cap surface, as shown in Fig. 39, this apparently negligible pore pressure difference near the drained limit may still lead to significant changes in the mechanical response in local areas.

Figure 37 shows the equivalent plastic strain contour for the punch loading at $0.002 \mathrm{~m} / \mathrm{day}$. At this stage, a clear localization zone is formed with plastic deformation developing inside. Discerning the effect of the cap model requires a closer look. To this end, we select four representative Gauss points, three of which are inside the localization band, while the fourth point is at the center of the circular punch, as shown in Fig. 38. The stress paths in meridional stress space $\sqrt{J_{2}^{\xi}}$ are recorded in Fig. 39a.

Among the four selected points, Points 1, 2 and 3 exhibit plastic deformation, while Point 4 , which is far away from the localization zone, remains in the elastic range. Moreover, Points 2 and 3, which are located inside the shearzone of the localization band, yield at the shear part of the yield surface. In particular, Point 1 , which is located near the circular punch tip, yields at the compactive (cap) portion of the yield surface. This feature is due to the high 


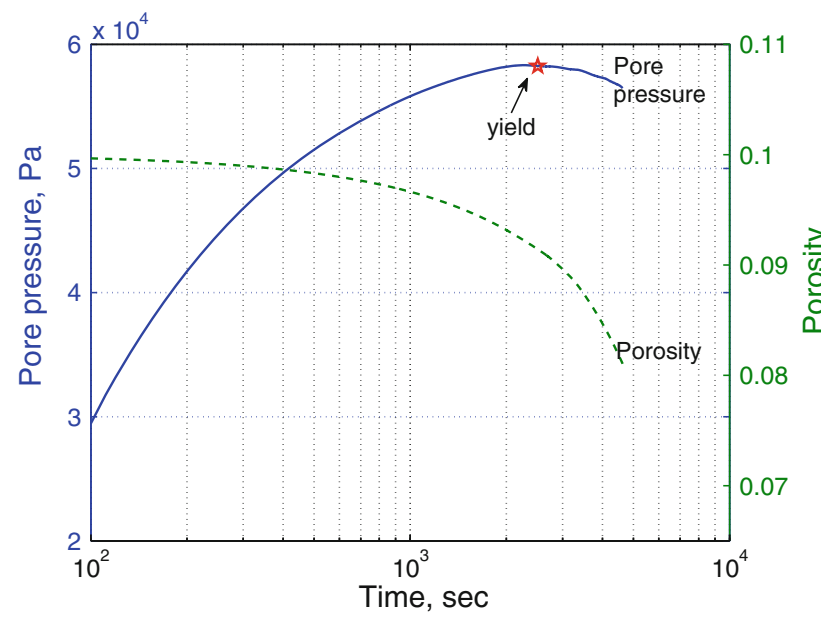

(a)

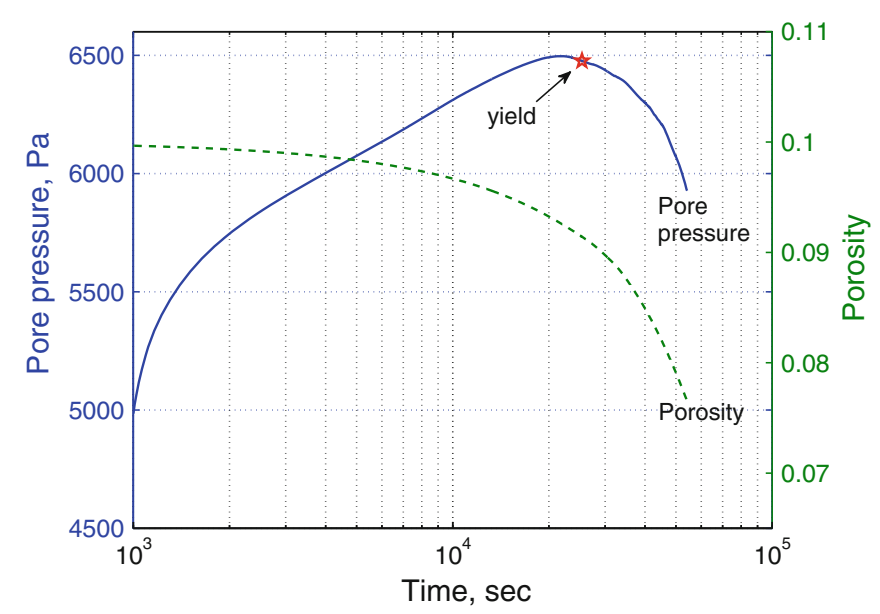

(b)

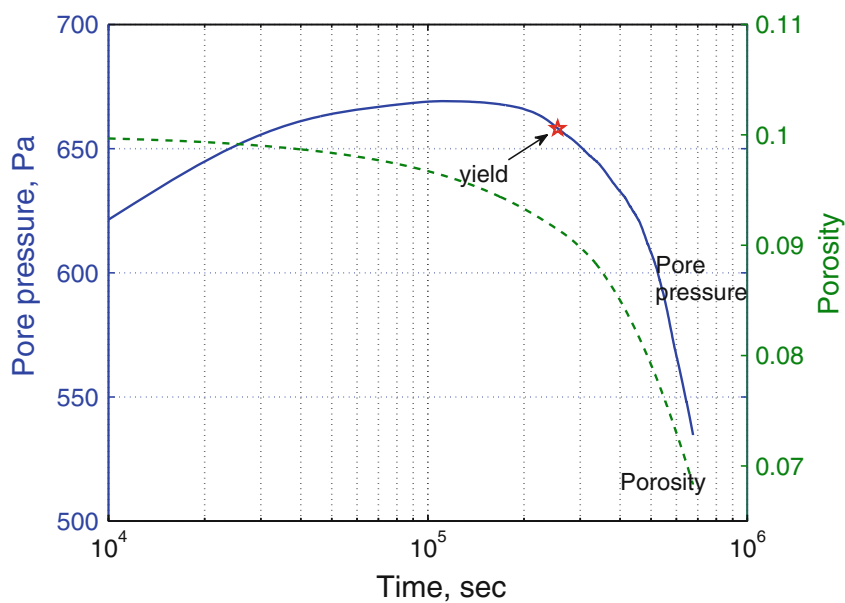

(c)

Fig. 36 Three-dimensional punch loading with associative cap plasticity. Pore pressure (left) and porosity (right) versus time (in log scale) for three different loading rates at edge of the circular punch (pt 1 in Fig. 38). Units in the figures are Pa. a $0.2 \mathrm{~m} / \mathrm{day}$ loading rate, b $0.02 \mathrm{~m} / \mathrm{day}$ loading rate, $\mathbf{c} 0.002 \mathrm{~m} /$ day loading rate

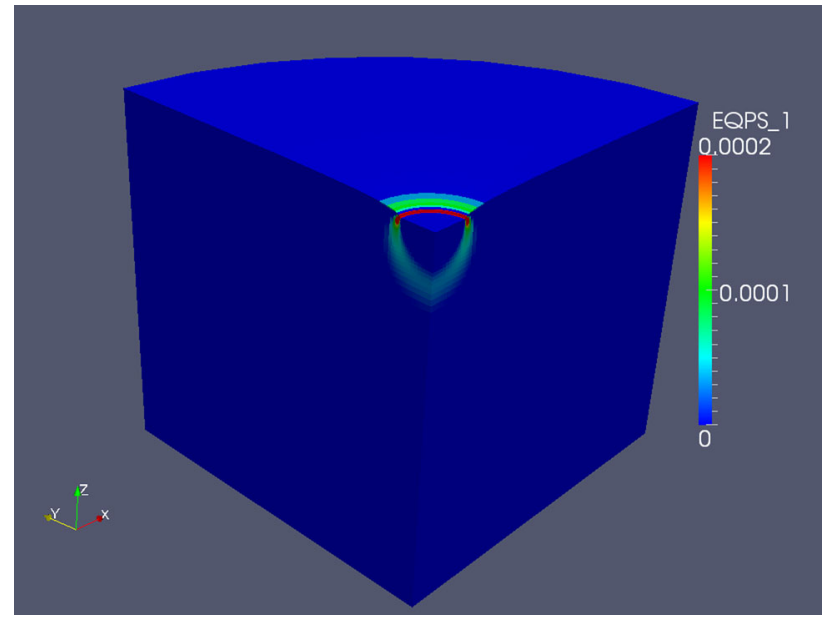

Fig. 37 Equivalent plastic strain contours of the rigid circular punch at $d=1.56 \times 10^{-2}$, with prescribed loading rate equals to 0.002 $\mathrm{m} /$ day hydrostatic stress at Point 1 . Collapsible porous media at such a high hydrostatic stress are likely to develop inelastic compaction due to the pore collapse. This mechanical characteristic is accurately captured by the cap plasticity model.

Figure $39 \mathrm{~b}$ shows the stress paths of Point 1 in meridional stress space $\sqrt{J_{2}}$ versus $I_{1}$. Although a small amount of plastic equivalent strain $(<0.0002)$ has been observed, the volumetric plastic strain is still dominant, as indicated by the porosity reduction in Fig. 36. This trend is consistent with the predicted stress path of Point 1 in Fig. 39b where the stress first lies on the cap part of the yield surface and approaches the cap part of the failure surface at the end of the loading.

In Fig. 40, we examine the porosity evolution for the four selected points, which illustrate the relation between the stress response and the underlying pore changes. We note that Point 1, which undergoes compactive 


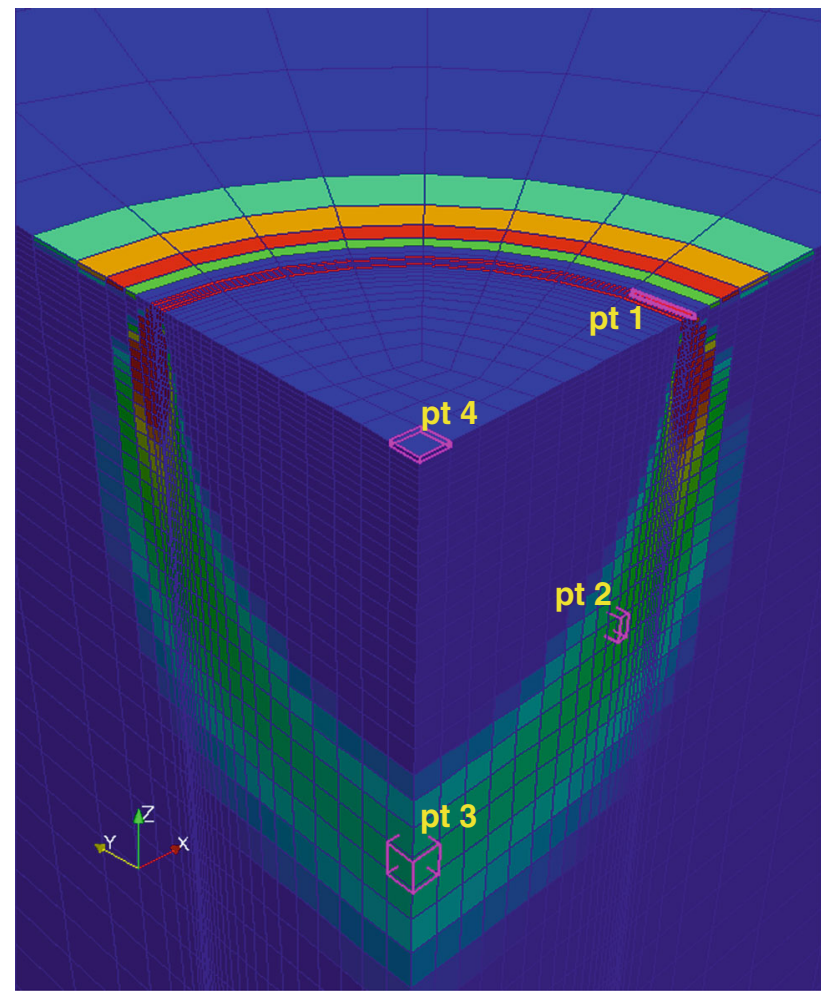

Fig. 38 Three-dimensional punch loading with associative cap plasticity. Equivalent plastic strain contour of the rigid circular punch at $d=1.56 \times 10^{-2} \mathrm{~m}$ with prescribed loading rate equals to 0.002 $\mathrm{m} / \mathrm{da}$. The stress paths of the four selected elements shown in this figure are recorded

deformation, experiences a rapid decrease in porosity compared with the other three points. Note that the porosity of Points 2 and 3 slightly decreases, even though both points are on the yield surface and experiencing plastic dilation. This porosity reduction is captured by the porosity evolution equation (52). According to (52), porosity reduction can be caused by the compression of the solid skeleton, shrinkages of pores and the volumetric expansion of the solid constituent. However, since pore pressure is small (as shown in Fig. 34) and the compressibilities of both constituents are low, the porosity reductions in Points 2 and 3 are mainly due to the elastic compression of the solid skeleton.

\section{Conclusion}

We examine the fully coupled hydro-mechanical response of water-saturated limestone subjected to loadings with various rates. Both non-associative and associative capplasticity models are incorporated into a stabilized monolithic mixed finite element method to simulate the fully coupled deformation-diffusion process of collapsible porous media. We found that hydro-mechanical couplings may impose various degrees of influence on the mechanical responses, depending on the loading rate, geometry of the domain, and the permeability of the porous medium. While this result is largely expected, we found that yielding of the punch tip often occurs on the cap surface, causing significant volumetric plastic strain accumulated. Despite the pore pressure build-up near the tip limiting the volumetric deformation, we still observe a considerable amount of compressive plastic strain under various combinations of loading rates and geometries. This accumulation of volumetric plastic strain leads to porosity and permeability reductions, hence affecting both the mechanical and hydraulic responses of the porous

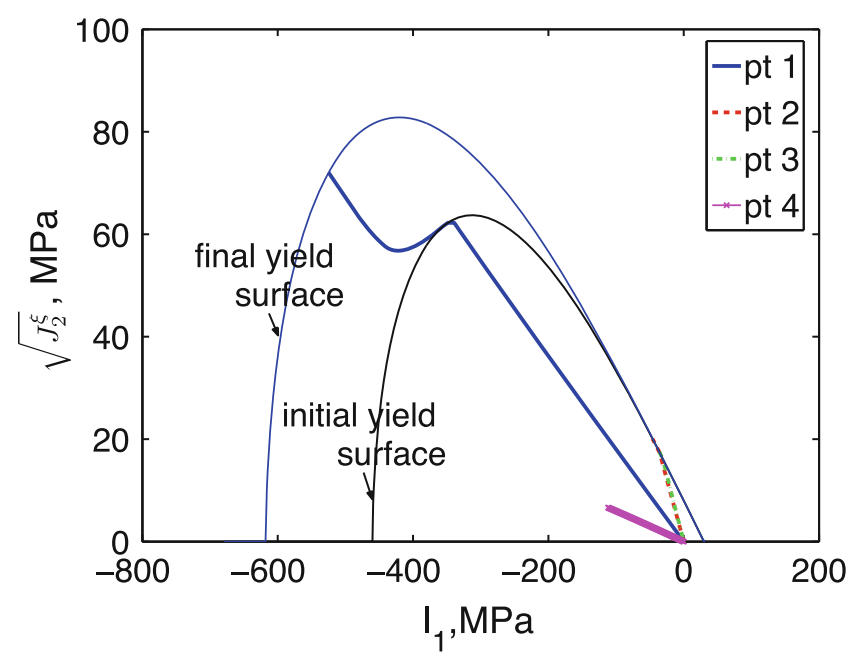

(a)

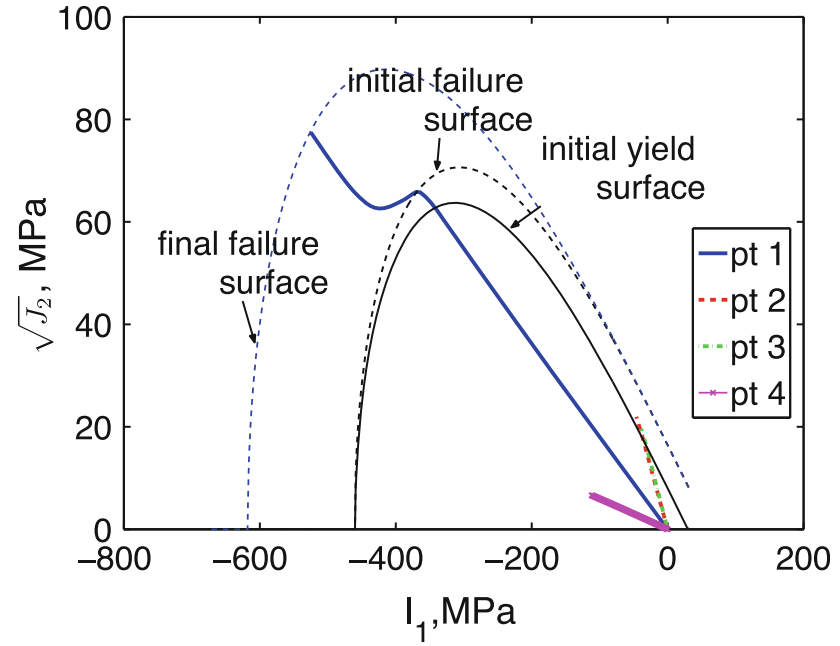

(b)

Fig. 39 Three-dimensional punch loading with associative cap plasticity. Stress path in the meridional stress space, a $\sqrt{J_{2}^{\xi}}$ versus $I_{1}$, and $\mathbf{b} \sqrt{J_{2}}$ versus $I_{1}$, for constant rate of $0.002 \mathrm{~m} /$ day. Locations of the four selected points are highlighted in Fig. 38 


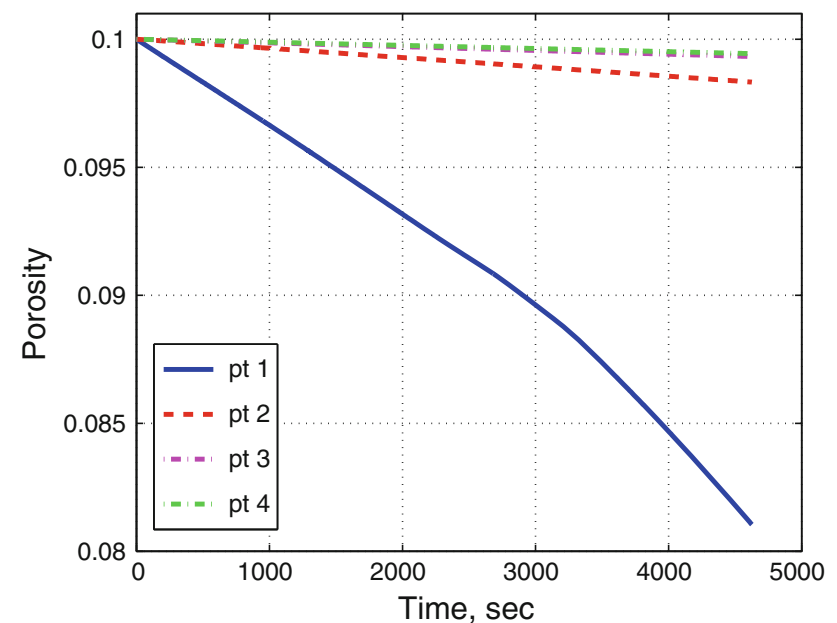

Fig. 40 Three-dimensional punch loading with associative cap plasticity. Porosity evolution for constant loading rate of 0.002 $\mathrm{m} /$ day. Location of the four selected points is shown in Fig. 38

rock. The numerical examples demonstrate that the cap surface of the plasticity model is important for determining the stability of the rock regardless of the drainage condition.

Acknowledgements Thanks are due to Professor Bernhard Schrefler for fruitful discussion. We are very grateful for the comprehensive reviews and insightful suggestions provided by the anonymous reviewers. Sandia National Laboratories is a multi-program laboratory managed and operated by Sandia Corporation, a wholly owned subsidiary of Lockheed Martin Corporation, for the US Department of Energy's National Nuclear Security Administration under contract DE-AC04-94AL85000.

\section{References}

1. Abellan M-A, de Borst R (2006) Wave propagation and localisation in a softening two-phase medium. Comput Methods Appl Mech Eng 195(37-40):5011-5019

2. Andrade JE, Borja RI (2006) Capturing strain localization in dense sands with random density. Int $\mathbf{J}$ Numer Methods Eng 67(11):1531-1564

3. Arnold DN (1990) Mixed finite element methods for elliptic problems. Comput Methods Appl Mech Eng 83(1-3):281-312

4. Babuška I (1973) The finite element method with Lagrangian multipliers. Numerische Mathematik 20:179-192

5. Bathe K-J (2001) The inf-sup condition and its evaluation for mixed finite element methods. Comput Struct 79(2):243-252

6. Bear J (1972) Dynamics of fluids in porous media. Elsevier Publishing Company, New York, NY

7. Belytschko T, Liu WK, Moran B (2000) Nonlinear finite elements for continua and structures. Wiley, West Sussex, England

8. Biot MA (1941) General theory of three dimensional consolidation. J Appl Phys 12(2):155-164

9. Bochev PB, Dohrmann CR, Gunzburger MD (2006) Stabilization of low-order mixed finite elements for the Stokes equations. SIAM J Numer Anal 44(1):82-101

10. Borja RI (2013) Plasticity: Modeling \& Computation. Springer, Berlin
11. Borja RI, Alarcón E (1995) A mathematical framework for finite strain elastoplastic consolidation part 1: Balance laws, variational formulation, and linearization. Comput Methods Appl Mech Eng 122(1-2):145-171

12. Brezzi F, Douglas J, Marini LD (1985) Two families of mixed finite elements for second order elliptic problems. Numerische Mathematik. 47:217-235

13. Chapelle D, Bathe KJ (1993) The inf-sup test. Comput Struct 47(4-5):537-545

14. Coussy O (2004) Poromehcanics. Wiley, West Sussex, England

15. de Souza Neto EA, Perić D, Owen DRJ (2008) Computational Methods for Plasticity. Wiley, Ltd, ISBN 9780470694626

16. DiMaggio FL, Sandler IS (1971) Material model for granular soils. J Eng Mech Div 97:935-950

17. Dolarevic S, Ibrahimbegovic A (2007) A modified three-surface elasto-plastic cap model and its numerical implementation. Comput Struct 85:419-430

18. Fossum AF, Fredrich JT (2000) Cap plasticity models and compactive and dilatant pre-failure deformation. In: Girard J, Liebman M, Breeds C, Doe T (eds) Pacific rocks 2000: rock around the rim. Seattle, WA, Taylor \& Francis pp 1169-1176

19. Foster CD, Regueiro RA, Fossum AF, Borja RI (2005) Implicit numerical integration of a three-invariant, isotropic/kinematic hardening cap plasticity model for geomaterials. Comput Methods Appl Mech Eng 194:5109-5138

20. Gamage K, Screaton E, Bekins B, Aiello I (2011) Permeabilityporosity relationships of subduction zone sediments. Mar Geol 279(1):19-36

21. Grueschow E, Rudnicki JW (2005) Elliptic yield cap constitutive modeling for high porosity sandstone. Int $\mathrm{J}$ Solids Struct 42:4574-4587

22. Jeremic B, Cheng Z, Taiebat M, Dafalias Y (2008) Numerical simulation of fully saturated porous materials. Int J Numer Anal Methods Geomech 32(13):1635-1660

23. Karaoulanis FE (2013) Implicit numerical integration of nonsmooth multisurface yield criteria in the principal stress space. Arch Comput Methods Eng 20:263-308

24. Ling HI, Liu H (2003) Pressure-level dependency and densification behavior of sand through generalized plasticity model. J Eng Mech 129(8):851-860

25. Moran B, Ortiz M, Shih CF (1990) Formulation of implicit finiteelement methods for multiplicative finite deformation plasticity. Int J Numer Methods Eng. 29(3):483-514

26. Mota A, Sun W, Ostien JT, Foulk JW, Long KN (2013) Liegroup interpolation and variational recovery for internal variables. Comput Mech (in press)

27. Nur A, Byerlee JD (1971) An exact effective stress law for elastic deformation of rock with fluids. J Geophys Res 76(26): 6414-6419

28. Prevost JH (1982) Nonlinear transient phenomena in saturated porous media. Comput Methods Appl Mech Eng. 30(1):3-18

29. Prevost JH, Høeg K (1976) Reanalysis of simple shear soil testing. Can Geotech J 13(4):418-429

30. Regueiro RA, Foster CD (2011) Bifurcation analysis for a ratesensitive, non-associative, three-invariant, isotropic/kinematic hardening cap plasticity model for geomaterials: Part I. small strain. Int J Numer Anal Methods Geomech 35:201-225

31. Rice JR, Cleary MP (1976) Some basic stress diffusion solutions for fluid-saturated elastic porous media with compressible constituents. Rev Geophys 14(2):227-241

32. Rudnicki JW, Rice JR (1975) Conditions for the localization of deformation in pressure-sensitive dilatant materials. J Mech Phys Solids 23(6):371-394

33. Sandler IS, Rubin D (1979) An algorithm and a modular subroutine for the cap model. Int J Numer Anal Methods Geomech 3:173-186 
34. Schulze O, Popp T, Kern H (2001) Development of damage and permeability in deforming rock salt. Eng Geol 61(2):163-180

35. Simo JC, Hughes TJR (1986) On the variational foundations of assumed strain methods. ASME J Appl Mech 53:51-54

36. Simon BR, Wu JS-S, Zienkiewicz OC, Paul DK (1986) Evaluation of $\mathrm{u}-\mathrm{w}$ and $\mathrm{u}-\pi$ finite element methods for the dynamic response of saturated porous media using one-dimensional models. Int J Numer Anal Methods Geomech. 10(5):461-482

37. Skempton AW (1954) The pore-pressure coefficient a and b. Geotechnique 4(4):143-147

38. Sloan SW, Abbo AJ, Sheng DC (2001) Refined explicit integration of elastoplastic models with automatic error control. Eng Computations 18:121-154

39. Stefanov YP, Chertov MA, Aidagulov GR, Myasnikov AV (2011) Dynamics of inelastic deformation of porous rocks and formation of localized compaction zones studied by numerical modeling. J Mech Phys Solids 59(11):2323-2340

40. Stevenson DL (1978) Salem limestone oil and gas production in the Keenville field, Wayne County, Illinois. Department of registration and education, state of Illinois

41. Sun W (2013) A unified method to predict diffuse and localized instabilities in sands. Geomech Geoeng Int J 8(2):65-75

42. Sun W, Andrade JE, Rudnicki JW, Eichhubl P (2011) Connecting microstructural attributes and permeability from $3 \mathrm{~d}$ tomographic images of in situ shear-enhanced compaction bands using multiscale computations. Geophys Res Lett 38(10):L10302

43. Sun W, Kuhn M, Rudnicki JW (2013a) A multiscale DEM-LBM analysis on permeability evolutions inside a dilatant shear band. Acta Geotech 8:465-480

44. Sun W, Ostien JT, Salinger A (2013b) A stabilized assumed deformation gradient finite element formulation for strongly coupled poromechanical simulations at finite strain. Int J Numer Anal Methods Geomech. doi:10.1002/nag.2161

45. Sun WC, Andrade JE, Rudnicki JW (2011) Multiscale method for characterization of porous microstructures and their impact on macroscopic effective permeability. Int J Numer Methods Eng 88(12):1260-1279

46. Swan CC, Seo YK (2000) A smooth, three-surface elasto-plastic cap model: rate formulation, integration algorithm and tangent operators. Research report, University of Iowa

47. Terzaghi K, Peck RB, Mesri G (1996) Soil mechanics in engineering practice. Wiley-Interscience, New York, NY

48. Tu X, Andrade Jose E, Chen Q (2009) Return mapping for nonsmooth and multiscale elastoplasticity. Comput Methods Appl Mech Eng. 198:2286-2296

49. Van Langen H, Vermeer PA (1991) Interface elements for singular plasticity points. Int J Numer Anal Methods Geomech 15(5):301-315

50. Wang B, Popescu R, Prevost JH (2004) Effects of boundary conditions and partial drainage on cyclic simple shear test resultsa numerical study. Int $\mathbf{J}$ Numer Anal Methods Geomech 28(10): 1057-1082

51. White JA, Borja RI (2008) Stabilized low-order finite elements for coupled solid-deformation/fluid-diffusion and their application to fault zone transients. Comput Methods Appl Mech Eng 197(49):4353-4366

52. Wriggers P (2010) Nonlinear finite element methods. SpringerVerlag, Berlin, Heidelberg

53. Xia K, Masud A (2006) New stabilized finite element method embedded with a cap model for the analysis of granular materials. J Eng Mech 132(3):250-259

54. Yang Y, Aplin AC (2007) Permeability and petrophysical properties of 30 natural mudstones. J Geophys Res Solid Earth (1978-2012) 112(B03206):1-14

55. Zhang HW, Schrefler BA (2001) Uniqueness and localization analysis of elastic plastic saturated porous media. Int J Numer Anal Methods Geomech 25(1):29-48 